\title{
Intramolecular 1,5- versus 1,6-hydrogen abstraction reaction promoted by alkoxyl radicals in pyranose and furanose models
}

\author{
Cosme G. Francisco, Raimundo Freire, Antonio J. Herrera, In'esP'erez-Mart' in and \\ Ernesto Su' arez*
}

Instituto de Productos Naturales y Agrobiolog' la del C.S.I.C., Carretera de La Esperanza 3, 38206 La Laguna, Tenerife, Spain

Received 11 May 2007; accepted 6 June 2007

Available online 12 June 2007

\begin{abstract}
The primary alkoxyl radical generated by reaction of 1-(2-hydroxyethyl)-glycosides with (diacetoxyiodo)benzene (DIB) and iodine can undergo regioselective intramolecular hydrogen abstraction (IHA) reactions to furnish four different dioxabicyclic systems derived from carbohydrates. The results strongly suggest that the regiocontrol and feasibility of the cyclisation are dependant not only on geometric and stereoelectronic factors, but also on polar and thermochemical factors. The correct selection of the substituents at the precursors can favour the 1,6-IHA against the 1,5-IHA pathway.
\end{abstract}

\section{Introduction}

Remote intramolecular free radical functionalisation of inactivated carbons via intramolecular hydrogen abstraction (IHA) from alkoxyl radicals has attracted considerable interest among synthetic organic chemists. ${ }^{-}$The (diacetoxyiodo)benzene (DIB) in the presence of iodine is a convenient reagent to generate alkoxyl radicals from alcohols in mild conditions and has been widely used in synthesis. Earlier reports from this laboratory have described a new methodology to obtain various bicyclic polyhydroxylated systems, starting from a carbohydrate with a primary alkoxyl radical attached to one, two, three and four carbon tethers extended from the $\mathrm{C} 1$ of the sugar, through an IHA reaction. $\underline{2}$ The 1,5hydrogen atom transfer (HAT) is by far the most common reaction and is particularly useful for the synthesis of tetrahydrofuran derivatives. $\frac{3,2 \mathrm{ff}}{1} 1,6$-HAT has also been frequently observed, but high yields are only obtained when the transferring hydrogen is bonded to an oxygen-substituted carbon.., 5 Among other factors such as geometry and stereoelectronic effects, the feasibility of the HAT reaction is dependant on polar effects and the $\mathrm{C}-\mathrm{H}$ dissociation bond energy (DBE) involved in the process. To some extent the $\mathrm{C}-\mathrm{H}$ DBE can be conditioned by the electron-donating ability of the substituents linked to the carbon atom. For instance, we can observe a decrease in the experimental $\mathrm{C}-\mathrm{H}$ DBE values ( $\mathrm{kcal} \mathrm{mol}^{-1}$ ) if the electron-donating ability of the $O$-substituent at the methane increases: $\mathrm{CH}_{3} \mathrm{C}-\mathrm{H}, 105$; $\mathrm{C}_{6} \mathrm{H}_{5} \mathrm{COOCH}_{2}-\mathrm{H}, 100.2$ and $\mathrm{CH}_{3} \mathrm{OCH}_{2}-\mathrm{H}, 93 .{ }^{6}$ In addition,

\footnotetext{
* Corresponding author. Tel.: +34 922 251004; fax: +34 922 260135; email: esuarez@ipna.csic.es
}

the polarity of the substituents can alter the regioselectivity of the hydrogen atom transfer promoted by a highly electrophilic alkoxyl radical. We have also found that either the absence of a substituent or the presence of alcohols protected with electron-withdrawing groups (EWGs) inhibits oxidation of the radical in the alkoxyl radical b-fragmentation reaction, allowing faster reaction with radical species present in the medium, typically capturing iodine. $\frac{7.8}{8}$

With all this in mind, the purpose of the present work was to investigate whether substituents could be used to control the reaction course in an IHA reaction. With this aim, we synthesised a number of carbohydrate models possessing a primary alkoxyl radical attached at $\mathrm{C} 1$ of pyranose and furanose $C$-glycosides by a two-carbon tether. In these models the alkoxyl radical may abstract hydrogen from two different carbon atoms: ${ }^{-}$from $\mathrm{C} 6$ and $\mathrm{C} 7$, in furanoses and pyranoses, respectively, through a seven-membered transition state (TS) and from $\mathrm{C} 4$ through a, in principle, more favourable sixmembered TS I ( $\underline{\text { Scheme 1) }}$.

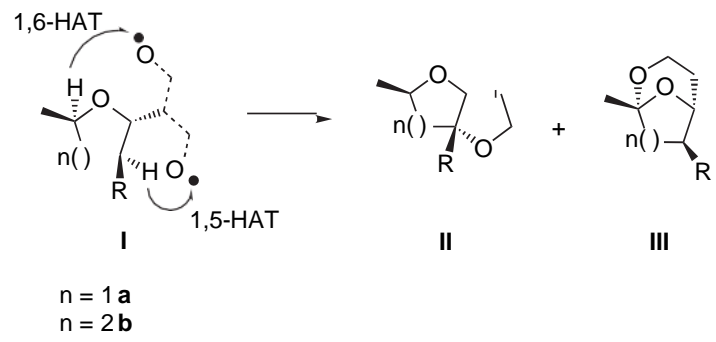

Scheme 1. Regioselectivity of the IHA reaction. 
It therefore offers an opportunity to study the regioselectivity of the process $(1,5$ - versus $1,6-$ HAT $) \underline{10}$ and at the same time to develop new methodology for the synthesis of four especially interesting dioxabicyclic systems: hexahydrofuro[3,2-b]furan IIa, hexahydro- $2 H$-furo[3,2-b]pyran IIb, 2,8-dioxabicyclo[3.2.1]octane IIIa and 2,9-dioxabicyclo[3.3.1]nonane IIIb. These bicycles are substructural units of many natural products. An example is azaspiracid, a marine toxin, which has units IIb and IIIb in its molecule. $\underline{11}$ Units $\mathrm{II}$ a and IIb are frequently related to ascorbic and zaragozic acid derivatives, respectively. $\frac{12}{}$

\section{Synthesis of substrates and results}

Preparation of the required octitols was accomplished following a well-established two-step protocol starting from suitably protected derivatives of L-fucose, L-rhamnose, Dmannose and 2-deoxy-D-arabinose. A Lewis acid-mediated $C$-glycosidation with allyltrimethylsilane afforded the non8 -enitols, in general with high stereoselectivity, $\underline{13,18}$ which upon subsequent ozonolysis followed by reductive workup with $\mathrm{NaBH}_{4}$ provided the alcohols 2, 4, 6, 8, 10, 13 and 17 in good yield (Scheme 2).
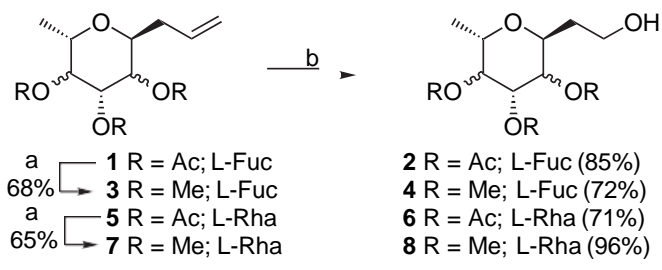

$2 \mathrm{R}=\mathrm{Ac} ; \mathrm{L}-\mathrm{Fuc}(85 \%)$

$4 \mathrm{R}=\mathrm{Me}$; L-Fuc $(72 \%)$

$6 \mathrm{R}=\mathrm{Ac} ; \mathrm{L}-\mathrm{Rha}(71 \%)$

$8 \mathrm{R}=\mathrm{Me}$; L-Rha $(96 \%)$
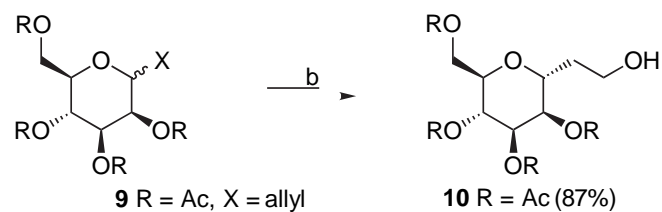

$\begin{array}{rl}C & 11 R=M e, X=O A c \\ 83 \% \square & 12 R=M e, X=\text { allyl }\end{array}$

$10 R=A c(87 \%)$
$13 R=\operatorname{Me}(79 \%)$

$\mathrm{RO}$
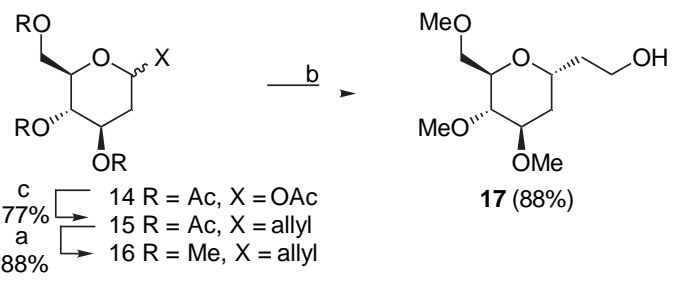

Scheme 2. Syntheses of 3,7-anhydrooctitols: (a) $\mathrm{KOH} / \mathrm{MeOH}$, rt followed by $\mathrm{NaH}, \mathrm{MeI}, \mathrm{DMF}, \mathrm{rt}$; (b) $\mathrm{O}_{3}, \mathrm{CH}_{2} \mathrm{Cl}_{2} / \mathrm{MeOH},-78^{\circ} \mathrm{C}$ and then $\mathrm{NaBH}_{4}$, $\mathrm{MeOH}, 0^{\circ} \mathrm{C}$; (c) ATMS, $\mathrm{BF}_{3} \mathrm{SOEt}_{2}, \mathrm{MeCN}, 0^{\circ} \mathrm{C} / \mathrm{rt}$.

We have also prepared three $C$-glycosides of the tetrahydrofuran series in order to extend this methodology for the synthesis of another two dioxabicyclic systems. The 3,6anhydro-heptitol 21 was prepared from the known ester $18^{2 \mathrm{c}}$ by changing the protecting groups and subsequent reduction of carboxyl moiety with $\mathrm{LiAlH}_{4}$. 3,6-Anhydrooctitol 23 was synthesised from 2,3:5,6-di- $O$ isopropylidene-D-man- nofuranose by radical $\mathrm{C}$ allylglycosidation as previously de- scribed $\underline{2 a}$ followed by ozonolysis with a reductive workup with $\mathrm{NaBH}_{4}$, which after suitable substitution of protecting groups yielded compound 26 (Scheme 3).<smiles>COC(=O)CC1OC(CO[AsH3])C2OC(C)(C)O[C@H]12</smiles>

18<smiles>COCC1OC(CCO)[C@H]2OCO[C@H]12</smiles>

21<smiles>C=CC[C@@H]1OC([C@H]2COC(C)(C)O2)C2OC(C)(C)OC21</smiles>

22

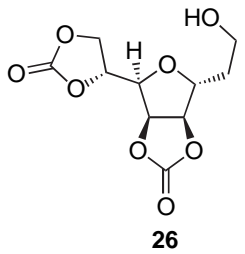

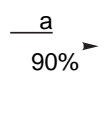

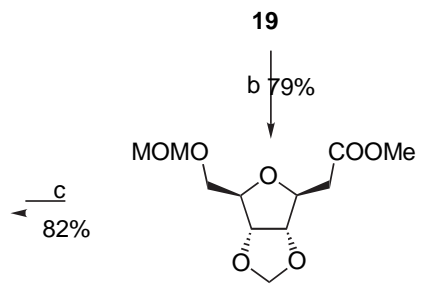

20
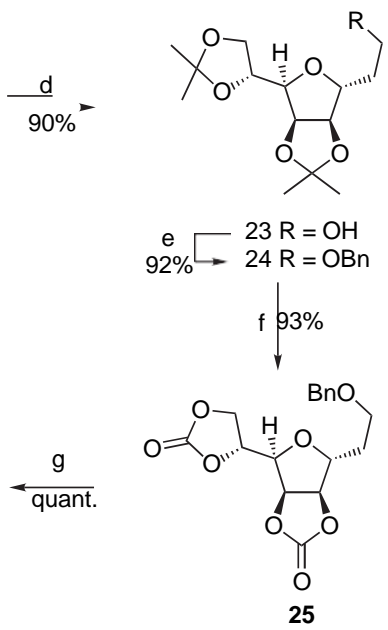

Scheme 3. Synthesis of 3,6-anhydroheptitols and 3,6-anhydrooctitols: (a) $\mathrm{I}_{2}, \mathrm{MeOH}$, reflux, $4 \mathrm{~h}$; (b) $(\mathrm{MeO})_{2} \mathrm{CH}_{2}, \mathrm{P}_{2} \mathrm{O}_{5}, \mathrm{CHCl}_{3}, \mathrm{rt}, 3 \mathrm{~h}$; (c) $\mathrm{LiAlH}_{4}$, THF, rt, $1 \mathrm{~h}$; (d) $\mathrm{O}_{3}, \mathrm{CH}_{2} \mathrm{Cl}_{2} / \mathrm{MeOH},-78^{\circ} \mathrm{C}$ and then $\mathrm{NaBH}_{4}, \mathrm{MeOH}$, $0{ }^{\circ} \mathrm{C}$; (e) $\mathrm{NaH}, \mathrm{BnBr}, \mathrm{DMF}$, rt; (f) acid resin Dowex $(50 \times 8), \mathrm{MeOH}, \mathrm{rt}$, $60 \mathrm{~h}$ followed by triphosgene, $\mathrm{Py}, \mathrm{CH}_{2} \mathrm{Cl}_{2},-70^{\circ} \mathrm{C} / \mathrm{rt}, 30 \mathrm{~min} ;(\mathrm{g}) \mathrm{H}_{2}$, $\mathrm{Pd}-\mathrm{C}, \mathrm{MeOH}, \mathrm{rt}, 8 \mathrm{~h}$.

The IHA reactions were performed under the oxidative conditions stated in Table 1, with (diacetoxyiodo)benzene and iodine in $\mathrm{CH}_{2} \mathrm{Cl}_{2}$ at room temperature and irradiation with two $80 \mathrm{~W}$ tungsten filament lamps.

Since the synthesis of the 2,9-dioxabicyclo[3.3.1] nonane system has never been reported from an IHA reaction, we decided to perform preliminary experiments to verify the feasibility of this methodology. Alcohols 2 and 4 derived from L-fucose were selected since $\mathrm{C} 4$ abstraction is stereochemically blocked and the reaction, in a conformationally restricted ${ }^{1} C$ pyranose ring, should proceed exclusively by abstraction of the hydrogen at C7 (entries 1 and 2). In both cases the reaction proceeded smoothly to give the expected dioxabicyclic products 27 and 28, respectively, in moderate yield.

In entry 3 we describe an IHA reaction over a $C$-glycoside derived from D-mannose 13. In this case, a restricted ${ }^{4} C_{1}$ conformation of the pyranose ring allows the hydroxyl radical at $\mathrm{C} 1$ to abstract the hydrogen atoms located at either the $\mathrm{C} 4$ equatorial or $\mathrm{C} 7$ axial position. The hexahydro- $2 \mathrm{H}$ - 
Table 1. IHA reaction of 3,7-anhydrooctitols and 3,6-anhydroheptitols ${ }^{\mathrm{a}}$

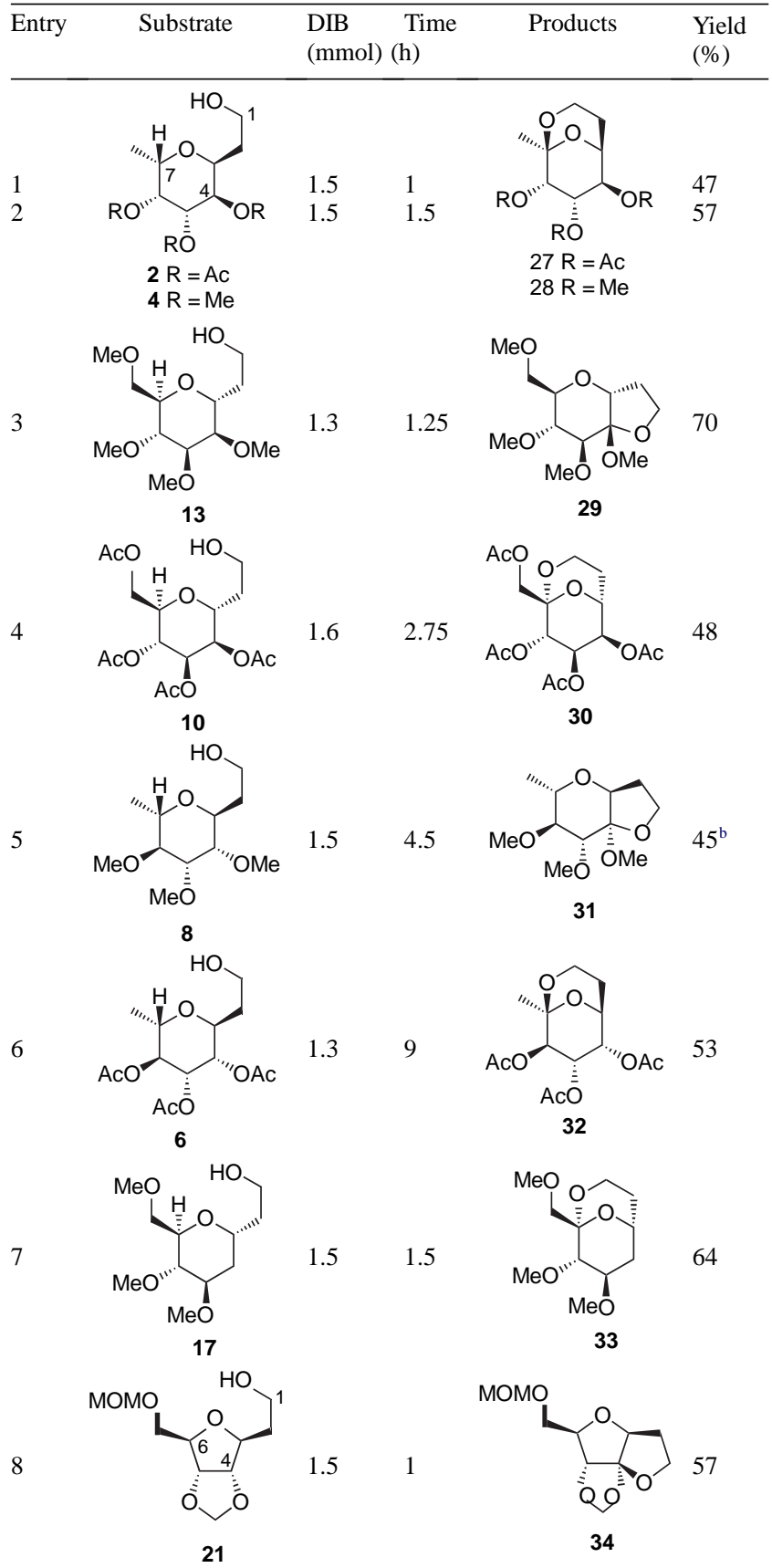

a Octitol or heptitol derivative $(1 \mathrm{mmol})$ in $\mathrm{CH}_{2} \mathrm{Cl}_{2}(25 \mathrm{~mL})$ containing DIB and iodine $(1 \mathrm{mmol})$ was irradiated with two $80 \mathrm{~W}$ tungsten filament lamps at room temperature.

b 2,8-Anhydro-1,7-dideoxy-3,4,5-tri- $O$-methyl-b-L-altro-oct-2-ulopyranose $(35,11 \%)$ was also obtained.

furo[3,2- $b]$ pyran derivative 29 , generated by the transfer of the hydrogen at $\mathrm{C} 4$, was formed exclusively.

The influence of the protective group at $\mathrm{C} 4$ was subsequently studied in entry 4 of Table 1 . The substitution of the methyl ether by an acetyl group (such as compound 10) affected the regiochemical course of the reaction; the $\mathrm{H}-\mathrm{C} 7$ was now transferred and the 2,9-dioxabicyclo[3.3.1]nonane derivative 30 was obtained instead. Although the yield was moderate, no other compounds could be detected in the reaction mixture.
The results obtained with two differentially protected 3,7anhydro-2,8-dideoxyoctitols 8 and 6 derived from L-rhamnose are shown in entries 5 and 6 . The IHA reaction proceeded analogously; the ester group at $\mathrm{C} 4$ inhibited the reaction originated by hydrogen transfer from this position and functionalisation occurred at C7 through a sevenmembered TS. The result obtained in the cyclisation of compound 8 merits a brief comment. Although the reaction gave mainly the expected product 31 corresponding to a $\mathrm{H}-\mathrm{C} 4$ hydrogen transfer, a minor product 35 , generated by a $\mathrm{H}-$ C7 hydrogen abstraction, was also obtained with a ratio 31/351/44:1. Interestingly, compound 13 reacts more selectively than its pseudo-enantiomer 8 with which the only difference is the 8-methoxy group. Probably the presence of a supplementary b-oxygen retards further the rate of hydrogen abstraction at $\mathrm{C} 7$, favouring the exclusive abstraction at C4. $\frac{14}{}$ A third minor compound 36 was isolated as a single stereoisomer when $\mathrm{NaHCO}_{3}$ was added to the reaction mixture, generated probably by a rarely observed double HAT as depicted in Scheme 4. $\frac{15}{} \mathrm{~A}$ possible explanation is that a preliminary $\mathrm{H}-\mathrm{C} 4$ hydrogen abstraction was followed by an intermolecular nucleophilic attack of an acetoxy group, coming from the reagent (DIB), to the transient oxycarbenium intermediate generated, maintaining the primary hydroxyl group enabled to carry out a second HAT and cyclisation at $\mathrm{C} 7$.

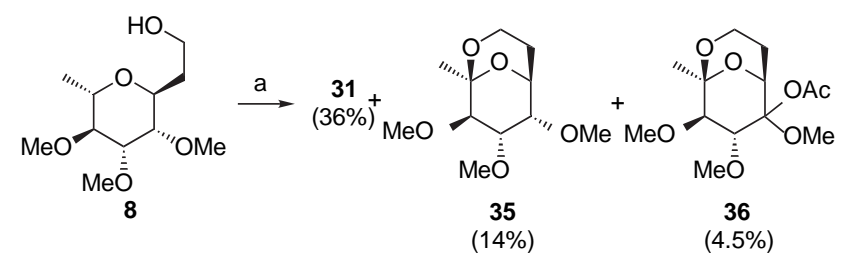

Scheme 4. (a) DIB (1.3 equiv), $\mathrm{I}_{2}$ (1 equiv), $\mathrm{NaHCO}_{3}(100 \%), \mathrm{CH}_{2} \mathrm{Cl}_{2}$, $100 \mathrm{~W}$ tungsten filament lamp, $1 \mathrm{~h}$.

Aware of the previously observed products of double IHA in our laboratory,,$\underline{2 a, b}$ where in the first instance a primary alkoxyl radical prefers a 1,5-HAT from a deoxygenated $\mathrm{CH}_{2}$ position instead of a 1,6-HAT from a pseudoanomeric position similar to our cases, we decided to synthesise the precursor 17 with a deoxygenated $\mathrm{C} 4$ position. For this sub- strate, 1,6-HAT dominated, furnishing exclusively product 33 in good yield instead of 1,5-HAT, which was expected to insert an iodine atom at position C4 (entry 7). For this pyranose model, the approximation of the alkoxyl radical to the $\mathrm{H}-\mathrm{C} 7$ atom in a respective 1,3-diaxial position is favoured with a smaller entropical penalty than in the case of a linear chain.

This IHA-cyclisation sequence was further extended to furanose models such as 21, 23 and 26. Model 21, derived from D-ribose, yielded smoothly the single expected compound 34 although with a quite tensioned tricyclic skeleton (entry 8). The use of acetal instead of methyl ethers as protecting group aimed to expand the scope of this methodology for further transformation of the products.

The D-mannofuranosyl derivatives 23 behaved similarly to the pyranose model 8 with poorer regiocontrol where $1,5-$ HAT dominated versus the 1,6-HAT, although both 
processes took place furnishing dioxabicyclic compounds 37 and 38 with a $67 \%$ yield in a ratio of (1.6:1) (Scheme 5). The regioselectivity decreased with respect to model 8 and no products of double HAT were observed. The yield of the re- action but not the products' ratio was optimised by neutral- ising the medium with a suspension of $\mathrm{NaHCO}_{3}$.<smiles>[R]C1OC[C@H]([C@H]2O[C@H](CCO)[C@H]3OC([R])O[C@@H]32)O1</smiles>

$23 \mathrm{R}=\mathrm{Me}_{2}$ $26 \mathrm{R}=\mathrm{O}$

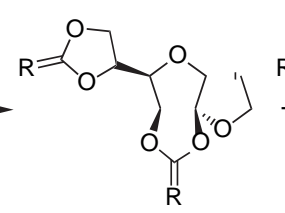

$37 \mathrm{R}=\mathrm{Me}_{2} 41 \%$ $39 \mathrm{R}=\mathrm{O} \quad 0 \%$

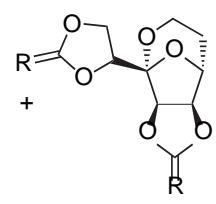

$38 \mathrm{R}=\mathrm{Me}_{2} 26 \%$ $40 \mathrm{R}=\mathrm{O} \quad 65 \%$
Scheme 5. 1,5- versus 1,6-IHA of mannofuranosyl derivatives. Reagent and conditions: (a) 23, DIB (2 equiv), $\mathrm{I}_{2}$ (1 equiv), $\mathrm{NaHCO}_{3}(30 \%)$, 2Xs@ tungsten filament lamps, rt, $1 \mathrm{~h}$; (b) 26, DIB (1.5 equiv), $\mathrm{I}_{2}$ ( 1 equiv), $\mathrm{CH}_{2} \mathrm{Cl}_{2}, 1 \times 80 \mathrm{~W}$ tungsten filament lamps, $\mathrm{rt}, 0.5 \mathrm{~h}$, and then $\mathrm{BF}_{3} \$ \mathrm{OEt}_{2}$ (1 equiv), $\mathrm{CH}_{2} \mathrm{Cl}_{2}, 0^{\circ} \mathrm{C}, 0.5 \mathrm{~h}$.

To our delight, we observed that upon switching the di- $O$ isopropylidene protecting group to carbonate, as depicted for model 26, the course of the reaction diverted exclusively to the 1,6-HAT at position C6 affording the product 40 in good yield. The carbonate group proved to be an EWG suf- ficient to inhibit the abstraction of $\mathrm{H}-\mathrm{C} 4$ to give compound

39. This time, the reaction procedure was modified, and a second step was necessary to complete the cyclisation stage with the addition of the Lewis acid $\left(\mathrm{BF}_{3} \$ \mathrm{OEt}_{2}\right)$. Otherwise, intermediate products of acetylation at $\mathrm{C} 6$ were isolated.

A mechanism to explain the observed regioselectivity of these IHA reactions is depicted in Scheme 6. When the substituent at $\mathrm{C} 4$ was an oxygenated electron-releasing group ( $\mathrm{R} 1 / 4$ ether or acetals), the alkoxyl radical I abstracted the hydrogen preferentially at this carbon atom through a sixmembered TS. The [3.3.0] and [4.3.0] bicycle, $n / 41$ and 2

(II), respectively, were subsequently formed, after oxidation at the C4 radical and intramolecular attack of the nucleophilic alcohol (path [a], models 8, 13, 21 and 23). Neverthe- less, the situation changed dramatically when $\mathrm{R}$ was

$$
\text { (b) }
$$

Scheme 6. Mechanism of the IHA reaction. hydrogen or an alcohol protected with an EWG (acetate or carbonate). The electrophilic alkoxyl radical abstracted the hydrogen exclusively on the electron-richer C6 or C7, $n$ 1/4 1 or 2, respectively, despite the less favourable sevenmembered TS, and the reaction went exclusively through path [b] to give the [3.3.1] or [3.2.1] bicycles (III) (models $6,10,17,26) . \underline{16}$ No compounds derived from abstraction of the hydrogen at the $\mathrm{C} 4$ position were detected in this case.

Intermolecular nucleophilic attack of anion acetate on the transient oxycarbenium cation intermediate is possible, the acetyl derivatives being isolable in some cases.

\section{Conclusions}

With these examples we have now demonstrated the possibility of using an EWG substituent to avoid the intramolecular functionalisation at a favoured position and trigger the reaction in a less favoured carbon atom. Indeed, the correct choice of the $\mathrm{C} 4$ substituent has been the switch to either 1,5-HAT or 1,6-HAT control in the reaction and hence to the specific synthesis of hexahydrofuro[3,2- $b]$ furan, hexahydro$2 H$-furo[3,2-b]pyran, 2,8-dioxabicyclo[3.2.1] octane or 2,9dioxabicyclo[3.3.1]nonane systems. $\frac{17}{}$ As observed, the reaction, which may be conceptually considered to be an intramolecular glycosidation is, in reality, a selective oxidation of specific carbons of the carbohydrate skeleton and constitute a mild procedure for the synthesis of protected uloses, which are not readily accessible by other methods.

\section{Experimental}

\subsection{General}

Melting points were determined with a hot-stage apparatus and are uncorrected. Optical rotations were measured at the sodium line at ambient temperature in $\mathrm{CHCl}_{3}$ solutions. IR spectra were measured as thin films on $\mathrm{NaCl}$ plate. $\mathrm{NMR}$ spectra were determined at $500 \mathrm{MHz}$ for ${ }^{1} \mathrm{H}$ and $125.7 \mathrm{MHz}$ for ${ }^{13} \mathrm{C}$ in $\mathrm{CDCl}_{3}$ unless otherwise stated, in the presence of TMS as internal standard. Mass spectra were determined at $70 \mathrm{eV}$. Merck silica gel $60 \mathrm{PF}(0.063-0.2 \mathrm{~mm})$ was used for column chromatography. Circular layers of $1 \mathrm{~mm}$ of Merck silica gel $60 \mathrm{PF}_{254}$ were used on a Chromatotron for centrifugally-assisted chromatography. Commercially available reagents and solvents were of analytical grade or were purified by standard procedures prior to use. All reactions involving air- or moisture-sensitive materials were carried out under a nitrogen atmosphere. The spray reagents for TLC analysis were conducted with $0.5 \%$ vanillin in $\mathrm{H}_{2} \mathrm{SO}_{4}-\mathrm{EtOH}(4: 1)$ and further heating until development of colour.

\subsection{Syntheses of precursors for cyclisation via IHA reaction}

4.2.1. 3,4,5-Tri-O-acetyl-2,6-anhydro-1,7-dideoxyL-glyc- ero-D-galacto-octitol (2). General procedure for reductive ozonolysis. A solution of 3,4,5-tri- $O$ acetyl-2,6-anhydro1,7,8,9-tetradeoxy-L-glycero-Dgalacto-non-8-enitol $13 \mathrm{a}$ 1

(434 mg, $1.38 \mathrm{mmol})$ in dry $\mathrm{CH} \mathrm{Cl}^{2}-2 \mathrm{MeOH}(50 \mathrm{~mL}, 1: 1)$ was cooled to $-78^{\circ} \mathrm{C}$ and ozone was introduced into the 
solution until it became blue. Then nitrogen was bubbled through the solution to expel excess of ozone, and the mixture was heated to $0^{\circ} \mathrm{C}$. Afterwards, $\mathrm{NaBH}_{4}$ (313 mg, $8.29 \mathrm{mmol}$ ) was added slowly and the solution stirred for $30 \mathrm{~min}$ at room temperature. The reaction mixture was then poured into water and extracted with $\mathrm{CH}_{2} \mathrm{Cl}_{2}$, dried over $\mathrm{Na}_{2} \mathrm{SO}_{4}$, and concentrated. Column chromatography (hexanes-EtOAc, 25:75) of the residue afforded the title alcohol 2 (375 mg, $1.18 \mathrm{mmol}, 85 \%)$ as a colourless oil: [a] -7.3 (c, 0.48); IR 3478, 2942, 1748, 1732, 1434, 1372,

1246, $1059 \mathrm{~cm}^{-1}$; ${ }^{1} \mathrm{H}$ NMR $1.11(3 \mathrm{H}, \mathrm{d}, J 1 / 46.3 \mathrm{~Hz}), 1.64$ $(1 \mathrm{H}, \mathrm{m}), 1.93(1 \mathrm{H}, \mathrm{m}), 1.96(3 \mathrm{H}, \mathrm{s}), 2.01(3 \mathrm{H}, \mathrm{s}), 2.10$ $(3 \mathrm{H}, \mathrm{s}), 3.69(2 \mathrm{H}, \mathrm{m}), 3.98(1 \mathrm{H}$, ddd, $J 1 / 41.9,6.5,6.5 \mathrm{~Hz})$, $4.34(1 \mathrm{H}$, ddd, $J 1 / 43.3,5.5,5.5 \mathrm{~Hz}), 5.13(1 \mathrm{H}, \mathrm{dd}, J 1 / 43.3$, $9.9 \mathrm{~Hz}), 5.22(1 \mathrm{H}, \mathrm{dd}, J / 41.5,5.4 \mathrm{~Hz}), 5.24(1 \mathrm{H}, \mathrm{dd}$, $J / 45.4,9.9 \mathrm{~Hz}) ;{ }^{13} \mathrm{C}$ NMR $15.8\left(\mathrm{CH}_{3}\right), 20.6\left(\mathrm{CH}_{3}\right), 20.7$ $\left(\mathrm{CH}_{3}\right), 20.7\left(\mathrm{CH}_{3}\right), 27.8\left(\mathrm{CH}_{2}\right), 59.8\left(\mathrm{CH}_{2}\right), 66(\mathrm{CH}), 68$ $(\mathrm{CH}), 68.5(\mathrm{CH}), 70.4(\mathrm{CH}), 70.6(\mathrm{CH}), 169.9(\mathrm{C}), 170.1$ (C), 170.5 (C); MS (EI) $\mathrm{m} / \mathrm{z}$ (rel intensity) $319\left(\mathrm{M}^{+}+\mathrm{H}, 1\right)$, $301(<1), 273(10)$; HRMS calcd for $\mathrm{C}_{14} \mathrm{H}_{23} \mathrm{O}_{8} 319.1393$, found 319.1359. Anal. Calcd for $\mathrm{C}_{14} \mathrm{H}_{22} \mathrm{O}_{8}$ : C, 52.82; $\mathrm{H}$, 6.97. Found: $\mathrm{C}, 52.72 ; \mathrm{H}, 7.25$.

4.2.2. 2,6-Anhydro-1,7,8,9-tetradeoxy-3,4,5-tri- $O$ methyl- L-glycero-D-galacto-non-8-enitol (3). A solution of 3,4,5-tri- $O$-acetyl-2,6-anhydro-1,7,8,9tetradeoxy-L-glycero- $\quad$ D-galacto-non-8-enitol 1 (3.6 g, $11.45 \mathrm{mmol})$ in $\mathrm{MeOH}(25 \mathrm{~mL})$ containing $\mathrm{KOH}(0.75 \mathrm{~g}$, $0.013 \mathrm{mmol}$ ) was stirred at room temperature for $1 \mathrm{~h}$. The reaction mixture was

then neutralised with Dowex $50 \times 8$ acid resin, filtered and concentrated in vacuum to afford an oily residue, which was used in the following reaction without purification. To a suspension of $\mathrm{NaH}(1.65 \mathrm{~g}, 68.72 \mathrm{mmol})$ in dry DMF (50 $\mathrm{mL}$ ) was added the crude triol previously obtained in $\mathrm{DMF}$ $(50 \mathrm{~mL})$ and the mixture stirred at $0{ }^{\circ} \mathrm{C}$ under nitrogen until hydrogen evolution had ceased. Then an excess of methyl iodide $(4.3 \mathrm{~mL}, 69.9 \mathrm{mmol})$ was added dropwise and stirring continued at room temperature for $2 \mathrm{~h}$. Excess reagent was destroyed by slow addition of $\mathrm{MeOH}$ and the solution poured into water and extracted with EtOAc. The organic extracts were dried over $\mathrm{Na}_{2} \mathrm{SO}_{4}$ and concentrated in vacuum. Column chromatography (hexanes-EtOAc, 70:30) of the residue afforded the title compound $3(1.81 \mathrm{~g}$, $7.87 \mathrm{mmol}, 68.6 \%)$ as a colourless oil: $[\mathrm{a}]_{\mathrm{D}}-86.7(\mathrm{c}$, 0.82); IR 2978, 2933, 2826, 1643, 1360, 1193, $1104 \mathrm{~cm}^{-1}$; ${ }^{1} \mathrm{H}$ NMR $1.27(3 \mathrm{H}, \mathrm{d}, J \mathrm{l} / 46.6 \mathrm{~Hz}), 2.30(1 \mathrm{H}, \mathrm{m}), 2.37(1 \mathrm{H}$, m), $3.44(3 \mathrm{H}, \mathrm{s}), 3.48(3 \mathrm{H}, \mathrm{s}), 3.49(3 \mathrm{H}, \mathrm{s}), 3.51(3 \mathrm{H}, \mathrm{m})$, $3.92(1 \mathrm{H}, \mathrm{dq}, J 1 / 46.5,3.3 \mathrm{~Hz}), 4.03(1 \mathrm{H}$, ddd, $J 1 / 49.1,5.3$, $3.8 \mathrm{~Hz}), 5.05(1 \mathrm{H}, \mathrm{d}, J / 410.2 \mathrm{~Hz}), 5.10\left(1 \mathrm{H}, \mathrm{dd}_{13} J 1 / 417.0\right.$, $1.5 \mathrm{~Hz}), 5.81(1 \mathrm{H}$, ddd, $J / 417.0,10.2,6.8 \mathrm{~Hz}) ;{ }^{13} \mathrm{C} \mathrm{NMR}$ $15.0\left(\mathrm{CH}_{3}\right), 31.9\left(\mathrm{CH}_{2}\right), 58.3\left(\mathrm{CH}_{3}\right), 58.5\left(\mathrm{CH}_{3}\right), 59.5$ $\left(\mathrm{CH}_{3}\right), 68.2(\mathrm{CH}), 70.0(\mathrm{CH}), 77.5(\mathrm{CH}), 77.6(\mathrm{CH}), 78.4$ $(\mathrm{CH}), 116.6\left(\mathrm{CH}_{2}\right), 135.2(\mathrm{CH})$; MS (EI) $\mathrm{m} / z$ (rel intensity) $230\left(\mathrm{M}^{+}, 21\right), 189$ (29); HRMS calcd for $\mathrm{C}_{12} \mathrm{H}_{22} \mathrm{O}_{4}$ 230.1518, found 230.1519. Anal. Calcd for $\mathrm{C}_{12} \mathrm{H}_{22} \mathrm{O}_{4}$ : C, 62.58; H, 9.63. Found: C, 62.49; H, 9.65.

4.2.3. 2,6-Anhydro-1,7-dideoxy-3,4,5-tri- $O$ methyl- L-glycero-D-galacto-octitol (4). Following the general procedure for ozonolysis, 2,6-anhydro-1,7,8,9tetradeoxy- 3,4,5-tri-O-methyl-L-glycero-D-galacto-non-8enitol 3 (1.4 g,

$6.08 \mathrm{mmol})$ afforded compound $4(1.025 \mathrm{~g}, 4.38 \mathrm{mmol}$, $72 \%)$ as a colourless oil: $[\mathrm{a}]_{\mathrm{D}}-65.5(c, 0.6)$; IR 3444,
2936, 2829, 1732, 1651, 1463, 1362, 1362, 1194, $1102 \mathrm{~cm}^{-1}$; ${ }^{1} \mathrm{H}$ NMR $1.28(3 \mathrm{H}, \mathrm{d}, J 1 / 46.6 \mathrm{~Hz}), 1.73(1 \mathrm{H}$, m), $1.94(1 \mathrm{H}, \mathrm{m}), 3.46(3 \mathrm{H}, \mathrm{s}), 3.49(3 \mathrm{H}, \mathrm{s}), 3.51(3 \mathrm{H}, \mathrm{s})$, $3.52(3 \mathrm{H}, \mathrm{m}), 3.77(2 \mathrm{H}, \mathrm{m}), 3.95(1 \mathrm{H}, \mathrm{dq}, J / 42.8,6.6 \mathrm{~Hz})$, $4.21(1 \mathrm{H}$, ddd, $J 1 / 43.6,9.9,9.9 \mathrm{~Hz}) ; \mathrm{C}$ NMR $15.4\left(\mathrm{CH}_{3}\right)$, $29.5\left(\mathrm{CH}_{2}\right), 58.3\left(\mathrm{CH}_{3}\right), 58.9\left(\mathrm{CH}_{3}\right), 59.9\left(\mathrm{CH}_{3}\right), 61.5$ $\left(\mathrm{CH}_{2}\right), 68.5(\mathrm{CH}), 70.8(\mathrm{CH}), 77.7(\mathrm{CH}), 77.9(\mathrm{CH}), 78.9$ (CH); MS (EI) $m / z$ (rel intensity) $235\left(\mathrm{M}^{+}+\mathrm{H},<1\right), 217$ $(<1), 202(<1), \quad 170(1)$; HRMS calcd for $\mathrm{C}_{11} \mathrm{H}_{22} \mathrm{O}_{5}$ 234.1467, found 234.1468. Anal. Calcd for $\mathrm{C}_{11} \mathrm{H}_{22} \mathrm{O}_{5}$ : C, 56.39; H, 9.46. Found: C, 56.20; H, 9.70.

4.2.4. 3,4,5-Tri-O-acetyl-2,6-anhydro-1,7-dideoxyL-glyc- ero-L-manno-octitol (6). Following the general procedure, 3,4,5-tri- $O$-acetyl-2,6-anhydro-1,7,8,9tetradeoxy-L-glycero- L-manno-non-8-enitol $13 \mathrm{~b} 5(500 \mathrm{mg}$, $1.591 \mathrm{mmol})$ afforded compound $6(361 \mathrm{mg}, 1.13 \mathrm{mmol}$, $71 \%)$ as a colourless oil: $[\mathrm{a}]_{\mathrm{D}}+0.6(c, 0.36)$; IR 3479 , 2940, 2889, 1746, 1372,

1228, $1048 \mathrm{~cm}^{-1} ;{ }^{1} \mathrm{H}$ NMR $1.21\left(3 \mathrm{H}, \mathrm{d}, J \frac{1}{4} 6.3 \mathrm{~Hz}\right), 1.77$ $(1 \mathrm{H}, \mathrm{m}), 1.98(3 \mathrm{H}, \mathrm{s}), 2.00(3 \mathrm{H}, \mathrm{m}), 2.04(3 \mathrm{H}, \mathrm{s}), 2.08$ (3H, s), 2.37 (1H, br d), 3.69-3.80 (3H, m), 4.08 (1H, ddd, $J 1 / 43.2,3.2,10.0 \mathrm{~Hz}), 4.99(1 \mathrm{H}, \mathrm{dd}, J 1 / 48.5,8.5 \mathrm{~Hz}), 5.14$ $5.17(2 \mathrm{H}, \mathrm{m}) ;{ }^{13} \mathrm{C}$ NMR $17.4\left(\mathrm{CH}_{3}\right), 20.6\left(\mathrm{CH}_{3}\right), 20.7$ $\left(\mathrm{CH}_{3}\right), 20.8\left(\mathrm{CH}_{3}\right), 31.1\left(\mathrm{CH}_{2}\right), 59.4\left(\mathrm{CH}_{2}\right), 68.4(\mathrm{CH}), 69$ $(\mathrm{CH}), 70.9(\mathrm{CH}), 71.4(\mathrm{CH}), 72.6(\mathrm{CH}), 169.8(\mathrm{C}), 170$ (C), 170.2 (C); MS (EI) $\mathrm{m} / z$ (rel intensity) $319\left(\mathrm{M}^{+}+\mathrm{H}, 1\right)$, 273 (2), 258 (1), 198 (32), 183 (48); HRMS calcd for $\mathrm{C}_{14} \mathrm{H}_{23} \mathrm{O}_{8} 319.1393$, found 319.1470. Anal. Calcd for $\mathrm{C}_{14} \mathrm{H}_{22} \mathrm{O}_{8}$ : C, 52.82; H, 6.97. Found: C, 52.56; H, 7.27.

4.2.5. 2,6-Anhydro-1,7,8,9-tetradeoxy-3,4,5-tri- $O$ methyl- L-glycero-L-manno-non-8-enitol (7). A solution of 3,4,5-tri- $O$-acetyl-2,6-anhydro-1,7,8,9tetradeoxy-L-glycero- L-manno-non-8-enitol 13b $5(11.5 \mathrm{~g}$, $36.59 \mathrm{mmol})$ in $\mathrm{MeOH}(200 \mathrm{~mL})$ containing $\mathrm{KOH}(6.0 \mathrm{~g}$, $150.0 \mathrm{mmol}$ ) was stirred at room temperature for $1 \mathrm{~h}$. The reaction mixture was

then neutralised with Dowex $50 \times 8$ acid resin, filtered and concentrated in vacuum to give an oil $(8.5 \mathrm{~g})$, which was used in the following reaction without purification. To a suspension of $\mathrm{NaH}(5.26 \mathrm{~g}, 219.5 \mathrm{mmol})$ in dry DMF $(350 \mathrm{~mL})$ was added the crude triol previously obtained in DMF (150 $\mathrm{mL}$ ) and the mixture stirred at $0{ }^{\circ} \mathrm{C}$ under nitrogen until all hydrogen evolution had ceased. Then an excess of methyl iodide $(15.9 \mathrm{~mL}, 256.1 \mathrm{mmol})$ was added dropwise and stirring continued at room temperature for $3 \mathrm{~h}$. Excess reagent was destroyed by slow addition of $\mathrm{MeOH}$ and the solution poured into water and extracted with EtOAc. The organic extracts were dried over $\mathrm{Na}_{2} \mathrm{SO}_{4}$ and concentrated in vacuum. Column chromatography (hexanes-EtOAc, 85:15) of the residue afforded the title compound $7(5.5 \mathrm{~g}, 23.88 \mathrm{mmol}$, $65.5 \%)$ as a colourless oil: [a $]_{\mathrm{D}}-8.2(c, 0.73)$; IR 3077, 2977, 2933, 2825, 1644, 1455, 1382, 1196, $1102 \mathrm{~cm}^{-1} ;{ }^{1} \mathrm{H}$ NMR $1.28(3 \mathrm{H}, \mathrm{d}, J \mathrm{l} / 46.3 \mathrm{~Hz}), 2.27(1 \mathrm{H}, \mathrm{m}), 2.42(1 \mathrm{H}, \mathrm{m})$, $3.16(1 \mathrm{H}, \mathrm{dd}, J / 47.7,7.7 \mathrm{~Hz}), 3.43(1 \mathrm{H}, \mathrm{m}), 3.43(3 \mathrm{H}, \mathrm{s})$, $3.45(1 \mathrm{H}, \mathrm{m}), 3.47(3 \mathrm{H}, \mathrm{s}), 3.52(3 \mathrm{H}, \mathrm{s}), 3.56(1 \mathrm{H}, \mathrm{m})$, 4.02 (1H, dq, $J 1 / 46.5,3.3 \mathrm{~Hz}), 5.09$ (1H, dd, $J 1 / 417.0$, $1.5 \mathrm{~Hz}), 5.12\left(1 \mathrm{H}, \mathrm{d}, J \frac{1}{4} 10.0 \mathrm{~Hz}\right), 5.79(1 \mathrm{H}, \mathrm{ddd}, J 1 / 417.0$, 10.2, $6.8 \mathrm{~Hz}) ;{ }^{13} \mathrm{C}$ NMR $17.8\left(\mathrm{CH}_{3}\right), 34.1\left(\mathrm{CH}_{2}\right), 57.6$ $\left(2 \times \mathrm{CH}_{3}\right), 60.2\left(\mathrm{CH}_{3}\right), 69.1(\mathrm{CH}), 71.9(\mathrm{CH}), 77.7(\mathrm{CH})$, $80(\mathrm{CH}), 81.8(\mathrm{CH}), 117.1\left(\mathrm{CH}_{2}\right), 134.2(\mathrm{CH})$; MS (EI) $\mathrm{m} / \mathrm{z}$ (rel intensity) 189 (44), 157 (139); HRMS calcd for $\mathrm{C}_{9} \mathrm{H}_{17} \mathrm{O}_{4}$ 189.1127, found 189.1155. Anal. Calcd for $\mathrm{C}_{12} \mathrm{H}_{22} \mathrm{O}_{4}$ : C, 62.58; H, 9.63. Found: C, 62.61; H, 9.64. 
4.2.6. 2,6-Anhydro-1,7-dideoxy-3,4,5-tri- $O$ methyl- L-glycero-L-manno-octitol (8). Following the general pro- cedure, 2,6-anhydro-1,7,8,9-tetradeoxy-3,4,5tri- $O$-methyl- $\quad$ L-glycero-L-manno-non-8-enitol $7(2.0 \mathrm{~g}$, $8.685 \mathrm{mmol})$ afforded compound $8(1.95 \mathrm{~g}, 8.32 \mathrm{mmol}$, $96 \%)$ as a colour-

less oil: $[\mathrm{a}]_{\mathrm{D}}-17.1(c, 0.34)$; IR 3453, 2935, 2828, 1725, $1642,1452,1383,1197,1099 \mathrm{~cm}^{-1} ;{ }^{1} \mathrm{H}$ NMR $1.28(3 \mathrm{H}, \mathrm{d}$, $J / 46.5 \mathrm{~Hz}), 1.67(1 \mathrm{H}, \mathrm{m}), 1.89(1 \mathrm{H}, \mathrm{m}), 3.17(1 \mathrm{H}, \mathrm{dd}$,

$\left.J^{1} / 48.1,8.1 \mathrm{~Hz}\right), 3.36\left(1 \mathrm{H}, \mathrm{dd}, J^{1} / 42.8,3.7 \mathrm{~Hz}\right), 3.41(3 \mathrm{H}, \mathrm{s})$, $3.45(3 \mathrm{H}, \mathrm{s}), 3.46(3 \mathrm{H}, \mathrm{s}), 3.47(1 \mathrm{H}, \mathrm{m}), 3.64(1 \mathrm{H}, \mathrm{dq}$, $J / 46.5,8.1 \mathrm{~Hz}), 3.73\left(2 \mathrm{H}, \mathrm{t}, J^{\mathrm{T}} / 46.0 \mathrm{~Hz}\right), 4.11(1 \mathrm{H}, \mathrm{ddd}$, $J 1 / 43.7,8.2,8.2 \mathrm{~Hz}) ;{ }^{C} \mathrm{C}$ NMR $17.5\left(\mathrm{CH}_{3}\right), 31.7\left(\mathrm{CH}_{2}\right)$, $57.6\left(\mathrm{CH}_{3}\right), 57.8\left(\mathrm{CH}_{3}\right), 59.7\left(\mathrm{CH}_{3}\right), 60.6\left(\mathrm{CH}_{2}\right), 69.3$ $(\mathrm{CH}), 70.7(\mathrm{CH}), 78.6(\mathrm{CH}), 79(\mathrm{CH}), 81.1(\mathrm{CH})$; MS (EI) $\mathrm{m} / \mathrm{z}$ (rel intensity) $234\left(\mathrm{M}^{+}, 1\right), 202$ (2), 189 (1); HRMS calcd for $\mathrm{C}_{11} \mathrm{H}_{22} \mathrm{O}_{5}$ 234.1467, found 234.1487. Anal. Calcd for $\mathrm{C}_{11} \mathrm{H}_{22} \mathrm{O}_{5}$ : C, 56.39; H, 9.46. Found: C, $56.34 ; \mathrm{H}, 9.45$.

4.2.7. 1,3,4,5-Tetra- $O$-acetyl-2,6-anhydro-7deoxy- D-glycero-D-manno-octitol (10). Following the general procedure, 1,3,4,5-tetra- $O$-acetyl-2,6-anhydro-7,8,9trideoxy- D-glycero-D-manno-non-8-enitol 18 $9(240 \mathrm{mg}$, $8.685 \mathrm{mmol})$ afforded compound $10(212 \mathrm{mg}, 0.56 \mathrm{mmol}$, $87 \%)$ as a col-

ourless oil: [a] $]_{\mathrm{D}}-3.1(c, 0.64)$; IR 3542, 2960, 2888, 1756, 1651, 1434, 1372, 1242, $1049 \mathrm{~cm}^{-1} ;{ }^{1} \mathrm{H}$ NMR $1.75(1 \mathrm{H}$, m), $1.92(1 \mathrm{H}, \mathrm{m}), 1.98(3 \mathrm{H}, \mathrm{s}), 2.01(3 \mathrm{H}, \mathrm{s}), 3.66(2 \mathrm{H}, \mathrm{m})$, $3.90(1 \mathrm{H}$, ddd, $J 1 / 42.8,7.5,7.5 \mathrm{~Hz}), 4.00\left(1 \mathrm{H}, \mathrm{dd}, J^{1} / 42.8\right.$, $12.2 \mathrm{~Hz}), 4.13\left(1 \mathrm{H}\right.$, ddd, $\left.J^{1} / 43.8,10.3,10.3 \mathrm{~Hz}\right), 4.41(1 \mathrm{H}$,

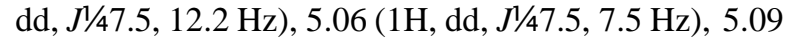
$\left(1 \mathrm{H}, \mathrm{dd}, J^{1 / 43} .8,3.8 \mathrm{~Hz}\right), 5.18\left(1 \mathrm{H}, \mathrm{dd}, J^{1 / 43.8}, 7.5 \mathrm{~Hz}\right) ;{ }^{13} \mathrm{C}$ NMR $21.0\left(2 \times \mathrm{CH}_{3}\right), 21.1\left(\mathrm{CH}_{3}\right), 21.2\left(\mathrm{CH}_{3}\right), 32.0\left(\mathrm{CH}_{2}\right)$, $59.6\left(\mathrm{CH}_{2}\right), 62.4\left(\mathrm{CH}_{2}\right), 67.7(\mathrm{CH}), 68.8(\mathrm{CH}), 70.7(\mathrm{CH})$, $71.7(\mathrm{CH}), 72.3(\mathrm{CH}), 170.2(\mathrm{C}), 170.5(2 \times \mathrm{C}), 171.2(\mathrm{C})$; MS (EI) $m / z$ (rel intensity) $377\left(\mathbf{M}^{+}-\mathrm{H},<1\right), 359(<1)$, $345(<1), 317$ (1), 214 (73); HRMS calcd for $\mathrm{C}_{16} \mathrm{H}_{25} \mathrm{O}_{10}$ 377.1448, found 377.1454. Anal. Calcd for $\mathrm{C}_{16} \mathrm{H}_{24} \mathrm{O}_{10}$ : C, 51.06; H, 6.43. Found: C, 50.70; H, 6.75 .

4.2.8. 2,6-Anhydro-7,8,9-trideoxy-1,3,4,5-tetra- $O$ methyl- D-glycero-D-manno-non-8-enitol (12). To a solution of 1 - $O$-acetyl-2,3,4,6-tetra- $O$-methyl-Dmannopyranose $\frac{19}{11}(104 \mathrm{mg}, 0.374 \mathrm{mmol})$ in dry MeCN $(1.5 \mathrm{~mL})$ were added allyltrimethylsilane $(0.176 \mathrm{~mL}, 1.122$ mmol) and $\mathrm{BF}_{3} \$ \mathrm{OEt}_{2}(0.114 \mathrm{~mL}, 0.935 \mathrm{mmol})$ at $0{ }^{\circ} \mathrm{C}$. After $30 \mathrm{~min}$ at room tem- perature, the reaction mixture was poured into ice-water and extracted with EtOAc. The organic extracts were dried over $\mathrm{Na}_{2} \mathrm{SO}_{4}$ and concentrated under reduced pressure. Column chromatography (hexanes-EtOAc, 1:1) of the residue af- forded the title compound 12 (82 mg, $0.31 \mathrm{mmol}, 83 \%)$ as a colourless oil: $[\mathrm{a}]_{\mathrm{D}}+15.6(c, 0.52) ;{ }^{1} \mathrm{H}$ NMR $2.33(1 \mathrm{H}, \mathrm{m}), 2.41(1 \mathrm{H}$, $\mathrm{m}), 3.37(3 \mathrm{H}, \mathrm{s}), 3.40(3 \mathrm{H}, \mathrm{s}), 3.41(1 \mathrm{H}, \mathrm{m}), 3.43(1 \mathrm{H}, \mathrm{m})$, $3.47(3 \mathrm{H}, \mathrm{s}), 3.47(3 \mathrm{H}, \mathrm{s}), 3.57(1 \mathrm{H}, \mathrm{m})$,

$3.58(2 \mathrm{H}, \mathrm{d}, J 1 / 44.7 \mathrm{~Hz}), 3.63(1 \mathrm{H}, \mathrm{m}), 4.02(1 \mathrm{H}, \mathrm{ddd}$,

$\left.J \frac{1}{4} 1.9,7.2,7.2 \mathrm{~Hz}\right), 5.07(1 \mathrm{H}, \mathrm{d}, J / 49.9 \mathrm{~Hz}), 5.10(1 \mathrm{H}, \mathrm{d}$, $J 1 / 417.1 \mathrm{~Hz}), 5.80\left(1 \mathrm{H}, \mathrm{dddd}, J \frac{1}{4} 7.3,7.3,10.0,17.1 \mathrm{~Hz}\right)$;

${ }^{13} \mathrm{C}$ NMR $34.1\left(\mathrm{CH}_{2}\right), 57.4\left(\mathrm{CH}_{3}\right), 57.6\left(\mathrm{CH}_{3}\right), 58.9\left(\mathrm{CH}_{3}\right)$, $59.6\left(\mathrm{CH}_{3}\right), 71.5\left(\mathrm{CH}_{2}\right), 71.8(\mathrm{CH}), 72.6(\mathrm{CH}), 76.4(\mathrm{CH})$, $77.1(\mathrm{CH}), 79.3(\mathrm{CH}), 117.1\left(\mathrm{CH}_{2}\right), 134.1(\mathrm{CH}) ; \mathrm{MS}(\mathrm{EI})$ $\mathrm{m} / \mathrm{z}$ (rel intensity) $219\left(\mathrm{M}^{+}-\mathrm{C}_{3} \mathrm{H}_{5}, 35\right), 187$ (63), 183 (15); HRMS calcd for $\mathrm{C}_{10} \mathrm{H}_{19} \mathrm{O}_{5}$ 219.1232, found 219.1218. Anal. Calcd for $\mathrm{C}_{13} \mathrm{H}_{24} \mathrm{O}_{5}$ : C, 59.98; H, 9.29. Found: C, 59.84; H, 9.14.
4.2.9. 2,6-Anhydro-7-deoxy-1,3,4,5-tetra- $O$ methyl- D-glycero-D-manno-octitol (13). Following the general procedure, 2,6-anhydro-7,8,9-trideoxy-1,3,4,5-tetra$O$-methyl- D-glycero-D-manno-non-8-enitol $12(500 \mathrm{mg}$, $1.921 \mathrm{mmol})$ afforded compound $13(398 \mathrm{mg}, 1.51 \mathrm{mmol}$, $79 \%)$ as a col- ourless oil: $[\mathrm{a}]_{\mathrm{D}}+19.2(c, 0.24)$; IR 3471, 2930, 1738, 1454, $1093 \mathrm{~cm}^{-1} ;{ }^{1} \mathrm{H}$ NMR $1.63(1 \mathrm{H}, \mathrm{m}), 1.84$ $(1 \mathrm{H}, \mathrm{m}), 3.29(3 \mathrm{H}, \mathrm{s}), 3.30(1 \mathrm{H}, \mathrm{m}), 3.32(1 \mathrm{H}, \mathrm{m}), 3.35$ $(3 \mathrm{H}, \mathrm{s}), 3.39$

$(3 \mathrm{H}, \mathrm{s}), 3.40(3 \mathrm{H}, \mathrm{s}), 3.45(1 \mathrm{H}, \mathrm{m}), 3.48\left(1 \mathrm{H}, \mathrm{dd}, J^{1} / 47.2\right.$, $10.0 \mathrm{~Hz}), 3.56\left(1 \mathrm{H}, \mathrm{dd}, J \frac{1}{4} 8.1,10.0 \mathrm{~Hz}\right), 3.66(1 \mathrm{H}, \mathrm{m}), 3.68$ $(2 \mathrm{H}, \mathrm{t}, J 1 / 45.7 \mathrm{~Hz}), 4.06(1 \mathrm{H}, \mathrm{ddd}, J 1 / 44.7,9.5,9.5 \mathrm{~Hz}) ;{ }^{13} \mathrm{C}$ NMR $31.7\left(\mathrm{CH}_{2}\right), 57.5\left(\mathrm{CH}_{3}\right), 58.2\left(\mathrm{CH}_{3}\right), 58.9\left(\mathrm{CH}_{3}\right)$, $59.4\left(\mathrm{CH}_{3}\right), 60.5\left(\mathrm{CH}_{2}\right), 71.2(\mathrm{CH}), 71.4\left(\mathrm{CH}_{2}\right), 72.3(\mathrm{CH})$, $76.6(\mathrm{CH}), 78.4(\mathrm{CH}), 78.9(\mathrm{CH})$; MS (EI) $\mathrm{m} / \mathrm{z}$ (rel intensity) $264\left(\mathrm{M}^{+},<1\right), 232$ (1), 219 (4), 200 (1), 187 (35); HRMS calcd for $\mathrm{C}_{12} \mathrm{H}_{24} \mathrm{O}_{6} 264.1573$, found 264.1530. Anal. Calcd for $\mathrm{C}_{12} \mathrm{H}_{24} \mathrm{O}_{6}$ : C, 54.53; H, 9.15. Found: C, 54.58; H, 9.18.

4.2.10. 6,7,9-Tri- $O$-acetyl-4,8-anhydro-1,2,3,5tetra- deoxy-D-manno-non-1-enitol (15). To a solution of com- pound $14^{20}(6.2 \mathrm{~g}, 18.6 \mathrm{mmol})$ in dry $\mathrm{MeCN}(60 \mathrm{~mL})$ were added allyltrimethylsilane $(9.3 \mathrm{~mL}$, $58.5 \mathrm{mmol})$ and $\mathrm{BF}_{3} \$ \mathrm{OEt}_{2}(6.2 \mathrm{~mL}, 48.9 \mathrm{mmol})$ at $0{ }^{\circ} \mathrm{C}$. After $1 \mathrm{~h}$ at room temperature, the reaction mixture was poured into ice-water and extracted with EtOAc. The organic extracts were dried over $\mathrm{Na}_{2} \mathrm{SO}_{4}$ and concentrated under reduced pressure. Col- umn chromatography (hexanes-EtOAc, 7:3) of the residue afforded the title compound $15(4.5 \mathrm{~g}, 14.3 \mathrm{mmol}, 77 \%)$ as a colourless oil: $[\mathrm{a}]_{\mathrm{D}}+38.0(c, 0.30) ; \mathrm{IR}\left(\mathrm{CCl}_{4}\right) 3080,2955,1748,1643$, 1433, 1367, 1231, $1050 \mathrm{~cm}^{-1} ;{ }^{1} \mathrm{H}$ NMR

$(400 \mathrm{MHz}) 1.85(1 \mathrm{H}$, ddd, $J / 44.8,8.9,13.6 \mathrm{~Hz}), 2.03(1 \mathrm{H}$, m), $2.05(3 \mathrm{H}, \mathrm{s}), 2.07(3 \mathrm{H}, \mathrm{s}), 2.08(3 \mathrm{H}, \mathrm{s}), 2.27(1 \mathrm{H}, \mathrm{m})$, $2.51(1 \mathrm{H}, \mathrm{m}), 3.91(1 \mathrm{H}, \mathrm{ddd}, J 1 / 43.3,6.7,6.7 \mathrm{~Hz}), 4.04$ $(1 \mathrm{H}, \mathrm{m}), 4.08\left(1 \mathrm{H}, \mathrm{dd}, J^{1} / 43.4,11.9 \mathrm{~Hz}\right), 4.38(1 \mathrm{H}, \mathrm{dd}$, $J 1 / 46.3,11.9 \mathrm{~Hz}), 4.86(1 \mathrm{H}, \mathrm{dd}, J / 47.3,7.3 \mathrm{~Hz}), 5.08-5.16$ $(3 \mathrm{H}, \mathrm{m}), 5.78(1 \mathrm{H}, \mathrm{dddd}, \mathrm{J} / 47.0,7.0,10.2,17.1 \mathrm{~Hz}) ;{ }^{13} \mathrm{C}$ NMR $(100.6 \mathrm{MHz}) 20.8\left(3 \times \mathrm{CH}_{3}\right), 32.0\left(\mathrm{CH}_{2}\right), 36.7$ $\left(\mathrm{CH}_{2}\right), 62.1(\mathrm{CH}), 68.7(\mathrm{CH}), 68.7\left(\mathrm{CH}_{2}\right), 69.8(\mathrm{CH}), 70.7$ $(\mathrm{CH}), 117.6\left(\mathrm{CH}_{2}\right), 133.9(\mathrm{CH}), 169.7(\mathrm{C}), 169.9(\mathrm{C})$, 170.6 (C); MS (EI) $\mathrm{m} / \mathrm{z}$ (rel intensity) (FAB) $337\left(\mathrm{M}^{+}+\mathrm{Na}\right.$, 7), $315\left(\mathrm{M}^{+}+\mathrm{H}, 75\right)$; HRMS calcd for $\mathrm{C}_{15} \mathrm{H}_{22} \mathrm{NaO}_{7}$ 337.1263, found 337.1258. Anal. Calcd for $\mathrm{C}_{15} \mathrm{H}_{22} \mathrm{O}_{7}$ : $\mathrm{C}$, 57.31; H, 7.05. Found: C, 57.40; H, 7.33.

4.2.11. 4,8-Anhydro-1,2,3,5-tetradeoxy-6,7,9-tri$O$-meth- yl-D-manno-non-1-enitol (16).

Compound $15 \quad(4.3 \mathrm{~g}$,

$13.7 \mathrm{mmol}$ ) was stirred in a $\mathrm{KOH}-\mathrm{MeOH} 3 \%$ solution (50 $\mathrm{mL}$ ) at room temperature for $12 \mathrm{~h}$. The reaction mixture was then neutralised with Dowex $50 \times 8$ acid resin, filtered and concentrated in vacuum to give an oily residue, which was used in the following reaction without purification. To a suspension of $\mathrm{NaH}(1.78 \mathrm{~g}, 74.2 \mathrm{mmol})$ in dry DMF (70 $\mathrm{mL}$ ) was added the crude tetrol previously obtained in DMF $(65 \mathrm{~mL})$ and the mixture stirred at $0^{\circ} \mathrm{C}$ under nitrogen until all hydrogen evolution had ceased. Then an excess of methyl iodide $(5.2 \mathrm{~mL}, 83.2 \mathrm{mmol})$ was added dropwise and stirring continued at room temperature for $3 \mathrm{~h}$. Excess reagent was destroyed by slow addition of $\mathrm{MeOH}$ and the solution poured into water and extracted with EtOAc. The organic extracts were dried over $\mathrm{Na}_{2} \mathrm{SO}_{4}$ and concentrated in vacuum. Column chromatography (hexanes-EtOAc, 6:4) of the residue afforded $16(2.8 \mathrm{~g}, 12.2 \mathrm{mmol}, 88 \%)$ as 
a colourless oil: [a $]_{\mathrm{D}}+42.2(c, 0.40)$; IR $\left(\mathrm{CCl}_{4}\right) 3078,2981$, 2929, 2822, 1642, 1446, 1377, 1193, $1107 \mathrm{~cm}^{-1} ;{ }^{1} \mathrm{H}$ NMR (400 MHz) $1.65(1 \mathrm{H}, \mathrm{ddd}, J 1 / 44.8,9.0,13.5 \mathrm{~Hz}), 1.93(1 \mathrm{H}$, ddd, $\left.J \frac{1}{4} 4.4,8.9,8.9 \mathrm{~Hz}\right), 2.23$ (1H, m), 2.45 (1H, m), 3.13 (1H, dd, Jl/47.1, $7.1 \mathrm{~Hz}), 3.39(3 \mathrm{H}, \mathrm{s}), 3.41(3 \mathrm{H}, \mathrm{s}), 3.48$ (1H, m), $3.49(3 \mathrm{H}, \mathrm{s}), 3.55(1 \mathrm{H}, \mathrm{m}), 3.62(1 \mathrm{H}, \mathrm{dd}, J 1 / 44.9$, $12.3 \mathrm{~Hz}), 3.65(1 \mathrm{H}, \mathrm{m}), 4.00(1 \mathrm{H}$, dddd, $J 1 / 44.7,4.7,7.4$, $7.4 \mathrm{~Hz}), 5.04-5.11(2 \mathrm{H}, \mathrm{m}), 5.78$ (1H, dddd, $J 1 / 46.9,6.9$, 10.1, 17.1 Hz); ${ }^{13} \mathrm{C}$ NMR (100.1 MHz) $32.0\left(\mathrm{CH}_{2}\right), 37.2$ $\left(\mathrm{CH}_{2}\right), 57.1\left(\mathrm{CH}_{3}\right), 59.3\left(\mathrm{CH}_{3}\right), 59.8\left(\mathrm{CH}_{3}\right), 70.4(\mathrm{CH})$, $71.8\left(\mathrm{CH}_{2}\right), 72.7(\mathrm{CH}), 78.2(\mathrm{CH}), 78.9(\mathrm{CH}), 117.2$ $\left(\mathrm{CH}_{2}\right), 134.9(\mathrm{CH})$; MS (EI) $\mathrm{m} / \mathrm{z}$ (rel intensity) (FAB) 253 $\left(\mathrm{M}^{+}+\mathrm{Na}, \quad 1\right), 231 \quad\left(\mathrm{M}^{+}+\mathrm{H}, 2\right)$; HRMS calcd for $\mathrm{C}_{12} \mathrm{H}_{22} \mathrm{NaO}_{4}$ 253.1416, found 253.1441. Anal. Calcd for $\mathrm{C}_{12} \mathrm{H}_{22} \mathrm{O}_{4}$ : C, 62.58; H, 9.63. Found: C, 62.41; H, 9.33. 4.2.12. 2,6-Anhydro-5,7-dideoxy-1,3,4-tri- $O$ methyl-D- manno-octitol (17). Following the general procedure for reductive ozonolysis, compound $16(2.4 \mathrm{~g}$, $10.6 \mathrm{mmol})$ af- forded compound $13(2.18 \mathrm{~g}, 9.3 \mathrm{mmol}$, $88 \%)$ as a colourless oil: $[\mathrm{a}]_{\mathrm{D}}+34.2(c, 0.26)$; IR 3638 , $3530,2929,2822,1455,1385,1191,1113 \mathrm{~cm}^{-1}$; ${ }^{1} \mathrm{H}$ NMR $1.43(1 \mathrm{H}, \mathrm{m}), 1.56(1 \mathrm{H}$,

ddd, $J 1 / 44.9,8.2,13.2 \mathrm{~Hz}), 1.73-1.86(2 \mathrm{H}, \mathrm{m}), 2.92(1 \mathrm{H}$, dd, $J / 46.4,6.4 \mathrm{~Hz}), 3.01(1 \mathrm{H}$, br s), $3.24(3 \mathrm{H}, \mathrm{s}), 3.26(3 \mathrm{H}$, s), $3.33(3 \mathrm{H}, \mathrm{s}), 3.34(1 \mathrm{H}, \mathrm{m}), 3.39(1 \mathrm{H}, \mathrm{dd}, J 1 / 43.6$, $10.2 \mathrm{~Hz}), 3.53(1 \mathrm{H}, \mathrm{dd}, J / 46.7,10.0 \mathrm{~Hz}), 3.57(2 \mathrm{H}, \mathrm{t}$, $J 1 / 46.0 \mathrm{~Hz}), 3.61(1 \mathrm{H}$, ddd, $J / 43.5,6.5,6.5 \mathrm{~Hz}), 3.99(1 \mathrm{H}$, dddd, $J 1 / 44.8,4.8,4.8,9.8 \mathrm{~Hz}) ;{ }^{13} \mathrm{C}$ NMR $32.4\left(\mathrm{CH}_{2}\right), 34.5$ $\left(\mathrm{CH}_{2}\right), 56.7\left(\mathrm{CH}_{3}\right), 58.8\left(\mathrm{CH}_{3}\right), 59.1\left(\mathrm{CH}_{3}\right), \quad 60.2\left(\mathrm{CH}_{2}\right)$, $68.6(\mathrm{CH}), 71.2\left(\mathrm{CH}_{2}\right), 72.1(\mathrm{CH}), 77.3(\mathrm{CH}), 78.2(\mathrm{CH})$; MS (FAB) $m / z$ (rel intensity) $257\left(\mathrm{M}^{+}+\mathrm{Na}, 5\right), 235\left(\mathrm{M}^{+}+\mathrm{H}\right.$, 100); HRMS calcd for $\mathrm{C}_{11} \mathrm{H}_{22} \mathrm{NaO}_{5}$ 257.1365, found 257.1340. Anal. Calcd for $\mathrm{C}_{11} \mathrm{H}_{22} \mathrm{O}_{5}$ : C, 56.39; $\mathrm{H}, 9.46$. Found: C, 56.27; H, 9.48.

4.2.13. Methyl 3,6-anhydro-2-deoxy-D-alloheptonate (19). A solution of $18^{21}(214 \mathrm{mg}, 0.44$ $\mathrm{mmol}$ ) and iodine (38 $\mathrm{mg}, 0.15 \mathrm{mmol})$ in methanol was refluxed for $4 \mathrm{~h}$. A sat- urated solution of $\mathrm{Na}_{2} \mathrm{~S}_{2} \mathrm{O}_{3}$ was added dropwise while the re- action mixture was stirred until complete reduction of the remaining iodine and the solvent was removed in vacuum. Purification of the residue by column chromatography $\left(\mathrm{CHCl}_{3}-\mathrm{MeOH}, 9: 1\right)$ gave compound $19(81.5 \mathrm{mg}$,

$0.40 \mathrm{mmol}, 90 \%)$ as a colourless oil: $[\mathrm{a}]_{\mathrm{D}}-2.3(c, 2.05)$; IR $\left(\mathrm{CCl}_{4}\right)$ 3360, 2931, 1732, 1652, 1442, 1285, 1108, $1040 \mathrm{~cm}^{-1}$; ${ }^{1} \mathrm{H}$ NMR $2.52(1 \mathrm{H}, \mathrm{dd}, J 1 / 48.4,15.4 \mathrm{~Hz}), 2.67$ (1H, dd, Jl/44.3, $15.5 \mathrm{~Hz}), 3.54(1 \mathrm{H}, \mathrm{dd}, J 1 / 44.5,12.0 \mathrm{~Hz})$, $3.63(1 \mathrm{H}, \mathrm{dd}, J 1 / 43.6,12.0 \mathrm{~Hz}), 3.67(3 \mathrm{H}, \mathrm{s}), 3.76(1 \mathrm{H}, \mathrm{dd}$, $J 1 / 46.0,6.0 \mathrm{~Hz}), 4.80(1 \mathrm{H}, \mathrm{ddd}, J 1 / 44.4,4.4,8.1 \mathrm{~Hz}), 3.95$ (1H, dd, Jl/44.4, 5.6 Hz), $4.09(1 \mathrm{H}, \mathrm{m}), 4.83\left(3 \mathrm{H}\right.$, br s); ${ }^{13} \mathrm{C}$ NMR $37.7\left(\mathrm{CH}_{2}\right), 50.7\left(\mathrm{CH}_{3}\right), 61.9\left(\mathrm{CH}_{2}\right), 71.0(\mathrm{CH}), 74.4$ $(\mathrm{CH}), 78.8(\mathrm{CH}), 84.8(\mathrm{CH}), 172.0(\mathrm{C})$; MS (EI) $\mathrm{m} / \mathrm{z}$ (rel intensity) (FAB) $229\left(\mathrm{M}^{+}+\mathrm{Na}, 100\right), 207\left(\mathrm{M}^{+}+\mathrm{H}, 100\right)$. Anal. Calcd for $\mathrm{C}_{8} \mathrm{H}_{14} \mathrm{O}_{6}$ : C, 46.60; H, 6.84. Found: C, 46.26; H, 7.22 .

4.2.14. Methyl $\begin{aligned} & \text { 3,6-anhydro-2-deoxy-7-O- } \\ & \text { (methoxy- } \\ & \text { methyl)-4,5-O-methylen-D-allo- }\end{aligned}$ (methoxy- methyl)-4,5-O-methylen-D-allo-
heptonate (20). To a solu- tion of compound $19(1.9 \mathrm{~g}$, $9.4 \mathrm{mmol})$ in dry $\mathrm{CHCl}_{3}(55 \mathrm{~mL})$ was added an excess of dimethoxymethane $(55 \mathrm{~mL}, 632.5 \mathrm{mmol})$, phosphorous pentoxide (28 g, $197 \mathrm{mmol})$ and stirred at room temperature under nitrogen atmosphere for $3 \mathrm{~h}$. The reaction mixture was cooled to
$0{ }^{\circ} \mathrm{C}$ and a saturated solution of $\mathrm{Na}_{2} \mathrm{CO}_{3}$ was added dropwise with vigorous stirring until the effervescence disappeared. The mixture was poured into water, extracted with $\mathrm{CH}_{2} \mathrm{Cl}_{2}$ and the organic layer was dried over $\mathrm{Na}_{2} \mathrm{SO}_{4}$ and concentrated in vacuum. Column chromatography of the residue (hexanes-EtOAc, 6:4) gave compound 20 (1.9g,

$7.4 \mathrm{mmol}, 79 \%)$ as a yellow oil: $[\mathrm{a}]_{\mathrm{D}}-0.8(c, 0.13)$; IR $\left(\mathrm{CCl}_{4}\right) 2955,1747,1438,1393,1343,1036 \mathrm{~cm}^{-1} ;{ }^{1} \mathrm{H}$ NMR (400 MHz) $2.72(2 \mathrm{H}, \mathrm{d}, J 1 / 46.3 \mathrm{~Hz}), 3.37(3 \mathrm{H}, \mathrm{s})$, 3.66-3.73 (2H, m), $3.71(3 \mathrm{H}, \mathrm{s}), 4.03(1 \mathrm{H}, \mathrm{ddd}, J 1 / 44.5$, $4.5,4.5 \mathrm{~Hz}), 4.23(1 \mathrm{H}$, ddd, $J 1 / 44.8,6.3,6.3 \mathrm{~Hz}), 4.49(1 \mathrm{H}$, dd, $J 1 / 44.8,6.9 \mathrm{~Hz}), 4.56(1 \mathrm{H}, \mathrm{dd}, J 1 / 44.8,6.9 \mathrm{~Hz}), 4.66$ $(2 \mathrm{H}, \mathrm{s}), 5.08(1 \mathrm{H}, \mathrm{s}), 5.13(1 \mathrm{H}, \mathrm{s}) ;{ }^{13} \mathrm{C} \mathrm{NMR}(100.6 \mathrm{MHz})$ $38.5\left(\mathrm{CH}_{2}\right), 52.2\left(\mathrm{CH}_{3}\right), 55.7\left(\mathrm{CH}_{3}\right), 68.1\left(\mathrm{CH}_{2}\right), 79.5$ (CH), $81.9(2 \times \mathrm{CH}), 84.1(\mathrm{CH}), 95.8\left(\mathrm{CH}_{2}\right), 97.1\left(\mathrm{CH}_{2}\right)$, 171.1 (C); MS (EI) $m / z$ (rel intensity) $261\left(\mathrm{M}^{+}-\mathrm{H}, 1\right), 247$ (1), 231 (6); HRMS calcd for $\mathrm{C}_{11} \mathrm{H}_{17} \mathrm{O}_{7}$ 261.0974, found 261.1002. Anal. Calcd for $\mathrm{C}_{11} \mathrm{H}_{18} \mathrm{O}_{7}$ : C, 50.38; H, 6.92. Found: C, 50.11; H, 7.10.

4.2.15.

(methoxymethyl)-4,5- $O$-methylen-D-allo-heptitol (21). To a solution of com- pound $20(992 \mathrm{mg}, 3.78$ mmol) in dry THF $(100 \mathrm{~mL})$ was added $\mathrm{LiAlH}_{4}(574 \mathrm{mg}$, $15.1 \mathrm{mmol}$ ) in small portions and stirred for $1 \mathrm{~h}$ at room temperature under nitrogen atmo- sphere. The excess hydride was destroyed by adding a satu- rated aqueous solution of $\mathrm{Na}_{2} \mathrm{SO}_{4}$, and the white salts were separated by filtration. The solvent was evaporated in vacuo and the residue purified by column chromatography (hex- anesEtOAc, 2:8) to afford compound 21 (723 mg,

$3.09 \mathrm{mmol}, 82 \%)$ as a colourless oil: $[\mathrm{a}]_{\mathrm{D}}-5.5(c, 0.20)$; IR 3637, 3549, 2941, 2884, 1424, 1214, 1154, 1081, 1043 $\mathrm{cm}^{-1}$; ${ }^{1} \mathrm{H}$ NMR $1.84-1.97(2 \mathrm{H}, \mathrm{m}), 2.32(1 \mathrm{H}$, br s, $\mathrm{OH}), 3.34(3 \mathrm{H}, \mathrm{s}), 3.66(1 \mathrm{H}, \mathrm{dd}, J 1 / 45.2,11.0 \mathrm{~Hz}), 3.71$ (1H, dd, J1/43.8, $11.0 \mathrm{~Hz}), 3.77\left(2 \mathrm{H}, \mathrm{t}, J \frac{1}{4} 5.7 \mathrm{~Hz}\right), 3.91-$ $3.96(2 \mathrm{H}, \mathrm{m}), 4.36(1 \mathrm{H}, \mathrm{dd}, J 1 / 45.5,6.9 \mathrm{~Hz}), 4.48(1 \mathrm{H}$, dd, J1/45.0, $6.9 \mathrm{~Hz}), 4.63(2 \mathrm{H}, \mathrm{s}), 5.02(1 \mathrm{H}, \mathrm{s}), 5.10(1 \mathrm{H}, \mathrm{s})$; ${ }^{13} \mathrm{C}$ NMR $35.5\left(\mathrm{CH}_{2}\right), 55.4\left(\mathrm{CH}_{3}\right), 60.1\left(\mathrm{CH}_{2}\right), 67.5$ $\left(\mathrm{CH}_{2}\right), 81.2(3 \times \mathrm{CH}), 84.0(\mathrm{CH}), 95.3\left(\mathrm{CH}_{2}\right), 96.6$ $\left(\mathrm{CH}_{2}\right) ; \mathrm{MS} \mathrm{m} / \mathrm{z}$ (rel intensity) $21^{+} 9\left(\mathrm{M}-\mathrm{CH}_{3}, 1\right), 203$ (7); HRMS calcd for $\mathrm{C}_{9} \mathrm{H}_{15} \mathrm{O}_{6} 219.087$, found 219.0827. Anal. Calcd for $\mathrm{C}_{10} \mathrm{H}_{18} \mathrm{O}_{6}$ : C, 51.27; H, 7.74. Found: C, 51.21; H, 8.09 .

4.2.16. 3,6-Anhydro-7-deoxy-1,2:4,5-di- $O$ isopropyl- idene-D-glycero-D-manno-octitol (23). Following the gen- eral procedure for reductive ozonolysis, compound $22 \underline{2 \mathrm{a}}(154 \mathrm{mg}, \quad 0.54 \mathrm{mmol})$ afforded compound 23 (140 mg,

$0.49 \mathrm{mmol}, 90 \%)$ as a colourless oil: $[\mathrm{a}]_{\mathrm{D}}-17(c, 0.6)$; IR 3552, 2987, 2873, 1350, $1215 \mathrm{~cm}^{-1}$; ${ }^{1} \mathrm{H}$ NMR 1.30 (3H, s), $1.34(3 \mathrm{H}, \mathrm{s}), 1.40(3 \mathrm{H}, \mathrm{s}), 1.46(3 \mathrm{H}, \mathrm{s}), 1.58(1 \mathrm{H}, \mathrm{m})$, $1.73(1 \mathrm{H}, \mathrm{m}), 2.20(1 \mathrm{H}$, br s, OH), $3.74(2 \mathrm{H}, \mathrm{m}), 3.81(1 \mathrm{H}$, dd, $J 1 / 44.2,6.9 \mathrm{~Hz}), 3.96(1 \mathrm{H}, \mathrm{dd}, J 1 / 44.9,8.7 \mathrm{~Hz}), 4.05$ (1H, dd, J1/46.4, 8.7 Hz), 4.23 (1H, ddd, $J 1 / 40.0,4.4$, $10.7 \mathrm{~Hz}), 4.36$ (1H, ddd, $J 1 / 46.5,6.5,6.5 \mathrm{~Hz}), 4.51(1 \mathrm{H}, \mathrm{dd}$, $J 1 / 40.0,6.0 \mathrm{~Hz}), 4.73(1 \mathrm{H}, \mathrm{dd}, J 1 / 43.8,6.0 \mathrm{~Hz}) ;{ }^{13} \mathrm{C} \mathrm{NMR}$ $24.6\left(\mathrm{CH}_{3}\right), 25.1\left(\mathrm{CH}_{3}\right), 26.0\left(\mathrm{CH}_{3}\right), 26.7\left(\mathrm{CH}_{3}\right), 32.5$ $\left(\mathrm{CH}_{2}\right), 60.3\left(\mathrm{CH}_{2}\right), 66.6\left(\mathrm{CH}_{2}\right), 73.3(\mathrm{CH}), 80.0(\mathrm{CH}), 80.5$ (CH), $83.1(\mathrm{CH}), 85.4(\mathrm{CH}), 109.0(\mathrm{C}), 112.6(\mathrm{C})$; MS (EI) $\mathrm{m} / \mathrm{z}$ (rel intensity) $273\left(\mathrm{M}^{+}-\mathrm{CH}_{3}, 50\right), 215$ (22), 101

(100); HRMS calcd for $\mathrm{C}_{13} \mathrm{H}_{21} \mathrm{O}_{6}$ 273.1338, found 273.1344. Anal. Calcd for $\mathrm{C}_{14} \mathrm{H}_{24} \mathrm{O}_{6}$ : C, 58.32; H, 8.39.

Found: C, 58.25; H, 8.58. 
4.2.17. 3,6-Anhydro-1-O-benzyl-2-deoxy-4,5:7,8di-O- isopropylidene-D-manno-octitol (24). Alcohol $23(1.64 \mathrm{~g}$,

$5.73 \mathrm{mmol})$ and $\mathrm{BnBr}(1.36 \mathrm{~mL}, 11.5 \mathrm{mmol})$ were dissolved in dry DMF $(15 \mathrm{~mL})$ under argon. $\mathrm{NaH}(60 \%$ in oil) $(458$ $\mathrm{mg}, 11.5 \mathrm{mmol}$ ) was added in portions at $0{ }^{\circ} \mathrm{C}$. After stirring for $2 \mathrm{~h}$ at room temperature, the reaction mixture was quenched with $\mathrm{MeOH}(5 \mathrm{~mL})$, poured into an aqueous sat. solution of $\mathrm{NH}_{4} \mathrm{Cl}$ and extracted with ether. The organic phase was separated, dried with anhydrous $\mathrm{Na}_{2} \mathrm{SO}_{4}$, filtered and concentrated under vacuum. Column chromatography (hexanes-EtOAc, 7:3) of the residue afforded compound $24(1.98 \mathrm{~g}, 5.24 \mathrm{mmol}, 92 \%)$ as a colourless oil: $[\mathrm{a}]_{\mathrm{D}}+18$ (c, 0.5, EtOAc); ${ }^{1} \mathrm{H}$ NMR $1.33(3 \mathrm{H}, \mathrm{s}), 1.37(3 \mathrm{H}, \mathrm{s}), 1.44$

$(3 \mathrm{H}, \mathrm{s}), 1.50(3 \mathrm{H}, \mathrm{s}), 1.73(2 \mathrm{H}, \mathrm{ddd}, J / 46.2,6.4,8.1 \mathrm{~Hz})$, $3.55(2 \mathrm{H}, \mathrm{dd}, J 1 / 46.5,6.5 \mathrm{~Hz}), 3.72(1 \mathrm{H}, \mathrm{dd}, J / 43.7$,

$7.7 \mathrm{~Hz}), 4.01(1 \mathrm{H}, \mathrm{dd}, J 1 / 44.5,8.7 \mathrm{~Hz}), 4.09(1 \mathrm{H}, \mathrm{dd}$, $\mathrm{J} / 46.4,8.7 \mathrm{~Hz}), 4.24(1 \mathrm{H}, \mathrm{dd}, \mathrm{J} / \mathrm{4} 7.4,7.4 \mathrm{~Hz}), 4.39(1 \mathrm{H}$, ddd, $J 1 / 44.4,6.2,7.7 \mathrm{~Hz}), 4.50(2 \mathrm{H}, \mathrm{s}), 4.59(1 \mathrm{H}, \mathrm{dd}$, $\left.J^{1} / 40.8,6.1 \mathrm{~Hz}\right), 4.75(1 \mathrm{H}, \mathrm{dd}, J 1 / 43.8,6.2 \mathrm{~Hz}), 7.27-7.38$ $(5 \mathrm{H}, \mathrm{m}) ;{ }^{13} \mathrm{C}$ NMR $25.1\left(\mathrm{CH}_{3}\right), 25.6\left(\mathrm{CH}_{3}\right), 26.5\left(\mathrm{CH}_{3}\right)$, $27.3\left(\mathrm{CH}_{3}\right), 67.2\left(\mathrm{CH}_{2}\right), 67.4\left(\mathrm{CH}_{2}\right), 73.5\left(\mathrm{CH}_{2}\right), 73.9$ $(\mathrm{CH}), 80.6(\mathrm{CH}), 81.1(\mathrm{CH}), 82.0(\mathrm{CH}), 85.9(\mathrm{CH}), 109.5$ (C), $112.9(\mathrm{C}), 127.9(3 \times \mathrm{CH}), 128.7(2 \times \mathrm{CH}), 138.7(\mathrm{C})$;

MS $\left(\mathrm{FAB}^{+}\right) \mathrm{m} / \mathrm{z}$ (rel intensity) $401\left(\mathrm{M}^{+}+\mathrm{Na}, 1\right), 379$ $\left(\mathrm{M}^{+}+\mathrm{H}, 4\right), 363$ (6); HRMS calcd for $\mathrm{C}_{21} \mathrm{H}_{31} \mathrm{O}_{6} 379.2121$, found 379.2117. Anal. Calcd for $\mathrm{C}_{21} \mathrm{H}_{30} \mathrm{O}_{6}: \mathrm{C}, 66.65 ; \mathrm{H}$, 7.99. Found: $\mathrm{C}, 66.70 ; \mathrm{H}, 7.91$.

4.2.18. 3,6-Anhydro-1-O-benzyl-2-deoxy-4,5:7,8di-O- oxomethylene-D-manno-octitol (25). Acid resin Dowex

$(50 \times 8)(29.0 \mathrm{~g}, 9$ equiv) was added to a solution of compound $24(1.95 \mathrm{~g}, 5.15 \mathrm{mmol})$ in methanol $(100 \mathrm{~mL})$ and stirred for $60 \mathrm{~h}$ at room temperature. The reaction mixture was filtered and the solvent removed under vacuum. The residue $(1.58 \mathrm{~g})$ and pyridine $(6.10 \mathrm{~g}, 77 \mathrm{mmol})$ dissolved in dry $\mathrm{CH}_{2} \mathrm{Cl}_{2}(21 \mathrm{~mL})$ was added slowly to a solution of triphosgene (3.36 g, $11.3 \mathrm{mmol})$ in $\mathrm{CH}_{2} \mathrm{Cl}_{2}(15 \mathrm{~mL})$ stirred at $-70^{\circ} \mathrm{C}$. After the addition was completed, the reaction mixture was allowed to warm up to room temperature. The resulting solution was poured into aqueous sat. solution of $\mathrm{NH}_{4} \mathrm{Cl}$ and extracted with $\mathrm{CH}_{2} \mathrm{Cl}_{2}$. The organic phase was washed with $1 \mathrm{~N} \mathrm{HCl}$, aqueous sat. solution of $\mathrm{NaHCO}_{3}$ and brine successively, dried over $\mathrm{Na}_{2} \mathrm{SO}_{4}$ and concentrated under vacuum. Column chromatography (hexanes-EtOAc, $4: 6)$ of the residue afforded compound 25 (1.68 $\mathrm{g}$,

$4.80 \mathrm{mmol}, 93 \%)$ as a white crystalline compound: $[\mathrm{a}]_{\mathrm{D}}$ +13 (c, 0.5, EtOAc); mp $118-120^{\circ} \mathrm{C}$ (EtOAc- $n$-pentane); ${ }^{1} \mathrm{H}$ NMR $1.81(2 \mathrm{H}, \mathrm{m}), 3.56\left(2 \mathrm{H}, \mathrm{dd}, J^{1} / 45.8,5.8 \mathrm{~Hz}\right), 4.13$ $(1 \mathrm{H}, \mathrm{dd}, J 1 / 42.9,5.6 \mathrm{~Hz}), 4.40(1 \mathrm{H}, \mathrm{dd}, J 1 / 46.4,8.7 \mathrm{~Hz})$, $4.46(1 \mathrm{H}, \mathrm{d}, J / 411.7 \mathrm{~Hz}), 4.49(1 \mathrm{H}, \mathrm{d}, J / 411.7 \mathrm{~Hz}), 4.52$ $(1 \mathrm{H}, \mathrm{dd}, J 1 / 48.6,8.6 \mathrm{~Hz}), 4.55(1 \mathrm{H}, \mathrm{dd}, J 1 / 47.0,7.0 \mathrm{~Hz})$, $4.93(1 \mathrm{H}$, ddd, $J 1 / 45.6,6.4,8.2 \mathrm{~Hz}), 5.14(1 \mathrm{H}, \mathrm{d}$, $\left.J^{1} / 49.8 \mathrm{~Hz}\right), 5.17\left(1 \mathrm{H}, \mathrm{d}, J^{1} / 49.8 \mathrm{~Hz}\right), 7.28-7.38(5 \mathrm{H}, \mathrm{m})$; ${ }^{13} \mathrm{C}$ NMR $30.9\left(\mathrm{CH}_{2}\right), 66.0\left(\mathrm{CH}_{2}\right), 66.5\left(\mathrm{CH}_{2}\right), 73.5(\mathrm{CH})$, $73.7\left(\mathrm{CH}_{2}\right), 79.2(\mathrm{CH}), 80.4(\mathrm{CH}), 83.0(\mathrm{CH}), 84.5(\mathrm{CH})$, $128.2(2 \times \mathrm{CH}, 128.4(\mathrm{CH}), 128.9(2 \times \mathrm{CH}), 138.0(\mathrm{C})$, $153.8(\mathrm{C}), 154.6(\mathrm{C})$; $\mathrm{MS}\left(\mathrm{FAB}^{+}\right) \mathrm{m} / \mathrm{z}$ (rel intensity) 373 $\left(\mathrm{M}^{+}+\mathrm{Na}, 7\right), 349\left(\mathrm{M}^{+}-\mathrm{H}, 11\right)$; HRMS calcd for $\mathrm{C}_{17} \mathrm{H}_{17} \mathrm{O}_{8}$ 349.0923, found 349.0915. Anal. Calcd for $\mathrm{C}_{17} \mathrm{H}_{18} \mathrm{O}_{8}: \mathrm{C}$, 58.28; H, 5.18. Found: C, 58.17; H, 5.45.

4.2.19. 3,6-Anhydro-2-deoxy-4,5:7,8-di- $O$ oxomethylene-

D-manno-octitol (26). Benzyl ether 25 (170 mg, 0.49 $\mathrm{mmol}$ ) was dissolved in $\mathrm{MeOH}(4 \mathrm{~mL})$ and Pd black $(17 \mathrm{mg})$ was added. The reaction vessel was purged from air and stirred for $8 \mathrm{~h}$ under a hydrogen atmosphere. Filtration through a Celite plug eluted with $\mathrm{MeOH}$ and concentration afforded the alcohol 26 (127 $\mathrm{mg}, 0.49 \mathrm{mmol}$, quant.) as a colourless

crystalline compound: $[\mathrm{a}]_{\mathrm{D}}-7(c, 0.4$, EtOAc); mp 116$117^{\circ} \mathrm{C}$ (EtOAc- $n$-pentane); ${ }^{1} \mathrm{H}$ NMR $1.80(2 \mathrm{H}$, ddd, $\left.J^{1} / 45.8,6.0,7.0 \mathrm{~Hz}\right), 3.82\left(2 \mathrm{H}, \mathrm{dd}, J^{1} / 45.7,5.7 \mathrm{~Hz}\right), 4.28$ $\left(1 \mathrm{H}, \mathrm{dd}, J^{1} / 43.6,4.9 \mathrm{~Hz}\right), 4.51\left(1 \mathrm{H}, \mathrm{dd}, J^{1} / 46.2,8.9 \mathrm{~Hz}\right)$, $4.60(1 \mathrm{H}, \mathrm{dd}, J / 48.4,8.4 \mathrm{~Hz}), 4.64(1 \mathrm{H}, \mathrm{dd}, J 1 / 47.3$, $7.3 \mathrm{~Hz}), 5.01\left(1 \mathrm{H}\right.$, ddd, $\left.J \frac{1}{4} 5.1,6.2,8.2 \mathrm{~Hz}\right), 5.20(1 \mathrm{H}, \mathrm{d}$, $J 1 / 46.6 \mathrm{~Hz}), 5.27\left(1 \mathrm{H}, \mathrm{dd}, J \frac{1}{43.7}, 7.1 \mathrm{~Hz}\right) ;{ }^{13} \mathrm{C} \quad \mathrm{NMR}$ $\left(\mathrm{CD}_{3} \mathrm{OD}\right) 33.7\left(\mathrm{CH}_{2}\right), 59.0\left(\mathrm{CH}_{2}\right), 67.8\left(\mathrm{CH}_{2}\right), 75.5(\mathrm{CH})$, $79.9(\mathrm{CH}), 82.2(\mathrm{CH}), 83.6(\mathrm{CH}), 86.1(\mathrm{CH}), 155.9(\mathrm{C})$, $156.8(\mathrm{C})$; $\mathrm{MS}\left(\mathrm{FAB}^{+}\right) \mathrm{m} / \mathrm{z}$ (rel intensity) $283\left(\mathrm{M}^{+}+\mathrm{Na}\right.$, 38), $261\left(\mathrm{M}^{+}+\mathrm{H}, 100\right)$; HRMS calcd for $\quad \mathrm{C}_{10} \mathrm{H}_{13} \mathrm{O}_{8}$ 261.0610, found 261.0602. Anal. Calcd for $\mathrm{C}_{10} \mathrm{H}_{13} \mathrm{O}_{8}$ : C, 46.16; H, 4.65. Found: C, 45.91; H, 4.82.

\subsection{IHA reaction of 3,7-anhydrooctitols and 3,6-anhydroheptitols}

4.3.1. General procedure. A solution of 3,7anhydroocti- tols or 3,6-anhydroheptitols $(1 \mathrm{mmol})$ in dry $\mathrm{CH}_{2} \mathrm{Cl}_{2}(25 \mathrm{~mL}$ ) containing (diacetoxyiodo)benzene (DIB) and iodine $(1 \mathrm{mmol})$ was irradiated with two $80 \mathrm{~W}$ tungsten filament lamps at room temperature under nitrogen until the reaction was completed. The reaction mixture was then poured into $10 \%$ aqueous $\mathrm{Na}_{2} \mathrm{~S}_{2} \mathrm{O}_{3}$ and extracted with $\mathrm{CH}_{2} \mathrm{Cl}_{2}$. The organic layer was dried and concentrated under vacuum. Chromatotron chromatography of the residue (hexanes-EtOAc mixtures) afforded the anhydrosugars. Equivalents of DIB used and reaction times are shown in Table 1 and Schemes 4 and 5.

4.3.2. Tri- $O$-acetyl-2,8-anhydro-1,7-dideoxy-b-Lgulo- oct-2-ulopyranose (27). Following the general procedure, precursor $2(33 \mathrm{mg}, 0.104 \mathrm{mmol})$ afforded the anhydrosugar

27 (15 mg, $0.05 \mathrm{mmol}, 47 \%)$ as a colourless oil: [a $]_{\mathrm{D}}-18.1$ (c, 0.16); IR 2978, 1732, 1715, 1372, 1234, $1064 \mathrm{~cm}^{-1}$; ${ }^{1} \mathrm{H}$ NMR $1.25(3 \mathrm{H}, \mathrm{s}), 1.90(1 \mathrm{H}, \mathrm{m}), 1.98(3 \mathrm{H}, \mathrm{s}), 2.04$ $(3 \mathrm{H}, \mathrm{s}), 2.11(1 \mathrm{H}, \mathrm{m}), 2.14(3 \mathrm{H}, \mathrm{s}), 3.76(1 \mathrm{H}, \mathrm{dd}, \mathrm{J} / \mathrm{4} 7.1$, $10.7 \mathrm{~Hz}), 3.87(1 \mathrm{H}$, ddd, $J / 44.7,11.3,11.3 \mathrm{~Hz}), 4.51(1 \mathrm{H}$, ddd, $\left.J^{\mathrm{l} / 43.2}, 5.9,9.6 \mathrm{~Hz}\right), 5.22\left(1 \mathrm{H}, \mathrm{d}, J^{1} / 44.1 \mathrm{~Hz}\right), 5.24$ $(1 \mathrm{H}, \mathrm{dd}, J / 45.9,10.5 \mathrm{~Hz}), 5.68(1 \mathrm{H}, \mathrm{dd}, J / 44.0,10.5 \mathrm{~Hz})$; ${ }^{13} \mathrm{C}$ NMR $(50.3 \mathrm{MHz}) 20.7\left(3 \times \mathrm{CH}_{3}\right), 22.2\left(\mathrm{CH}_{3}\right), 23.6$ $\left(\mathrm{CH}_{2}\right), 55.9\left(\mathrm{CH}_{2}\right), 65.9(\mathrm{CH}), 67.1(\mathrm{CH}), 67.8(\mathrm{CH}), 71.4$ (CH), 98.3 (C), 169.7 (C), 169.9 (C), 170.1 (C); MS (EI) $\mathrm{m} / \mathrm{z}$ (rel intensity) $316\left(\mathrm{M}^{+}, 1\right), 257$ (17), 214 (5), 196 (16); HRMS calcd for $\mathrm{C}_{14} \mathrm{H}_{20} \mathrm{O}_{8} 316.1158$, found 316.1175 . Anal. Calcd for $\mathrm{C}_{14} \mathrm{H}_{20} \mathrm{O}_{8}$ : C, 53.16; H, 6.37. Found: C, 53.06; H, 6.70 .

4.3.3. 2,8-Anhydro-1,7-dideoxy-3,4,5-tri- $O$ methyl-b- L-gulo-oct-2-ulopyranose (28). Following the general procedure, precursor 4 (131 mg, $0.559 \mathrm{mmol})$ afforded the anhydrosugar $28(75 \mathrm{mg}, 0.32$ $\mathrm{mmol}, 57 \%)$ as a colourless oil: [a] $]_{\mathrm{D}}-30.5(c, 0.59)$; IR 2936, 2826, 1462, 1377, 1223, $1102,1074 \mathrm{~cm}^{-1} ;{ }^{1} \mathrm{H}$ NMR $1.33(3 \mathrm{H}, \mathrm{s}), 1.76(1 \mathrm{H}$, m), $2.06(1 \mathrm{H}, \mathrm{m}), 3.37(1 \mathrm{H}, \mathrm{d}, J 1 / 42.8 \mathrm{~Hz}), 3.4(3 \mathrm{H}, \mathrm{s}), 3.5$ $(3 \mathrm{H}, \mathrm{s}), 3.56(3 \mathrm{H}, \mathrm{s}), 3.60(1 \mathrm{H}, \mathrm{dd}, J \mathrm{r} / 43.3,9.9 \mathrm{~Hz}), 3.62$ $(1 \mathrm{H}, \mathrm{d}, J / 46.1 \mathrm{~Hz}), 3.79\left(1 \mathrm{H}, \mathrm{dd}, J^{1} / 43.3,9.9 \mathrm{~Hz}\right), 3.84(1 \mathrm{H}$, ddd, $J 1 / 44.3,9.9,9.9 \mathrm{~Hz}$ ), 4.37 (1H, ddd, $J 1 / 43.8,6.1$, 
$10.0 \mathrm{~Hz}) ;{ }^{13} \mathrm{C}$ NMR $22.6\left(\mathrm{CH}_{3}\right), 23.3\left(\mathrm{CH}_{2}\right), 55.9\left(\mathrm{CH}_{2}\right)$, $58.1\left(\mathrm{CH}_{3}\right), 58.4\left(\mathrm{CH}_{3}\right), 61.6\left(\mathrm{CH}_{3}\right), 66.3(\mathrm{CH}), 77(\mathrm{CH})$, $79(\mathrm{CH}), 80.2(\mathrm{CH}), 99.5(\mathrm{C})$; MS (EI) $\mathrm{m} / \mathrm{z}$ (rel intensity) $231\left(\mathrm{M}^{+}, \quad<1\right), 203$ (4), 187 (4); HRMS calcd for $\mathrm{C}_{11} \mathrm{H}_{19} \mathrm{O}_{5} 231.1232$, found 231.1291. Anal. Calcd for $\mathrm{C}_{11} \mathrm{H}_{20} \mathrm{O}_{5}$ : C, 56.88; H, 8.68. Found: C, 56.54; H, 9.02. 4.3.4. Methyl 3,7-anhydro-2-deoxy-5,6,8-tri- $O$ methyl-a- D-manno-oct-4-ulofuranoside (29). Following the general procedure, precursor 13 (249 mg, $0.942 \mathrm{mmol})$ afforded the anhydrosugar 29 (174 $\mathrm{mg}, 0.66$ mmol, $70 \%)$ as a colour- less oil: $[\mathrm{a}]_{\mathrm{D}}+13.3(c, 0.42)$; IR 2933, 2828, 1740, 1456, 1194, $1106 \mathrm{~cm}^{-1} ;{ }^{1} \mathrm{H}$ NMR 1.87 (1H, m), $2.27(1 \mathrm{H}, \mathrm{m})$,

$3.17(1 \mathrm{H}, \mathrm{dd}, J 1 / 42.6,6.9 \mathrm{~Hz}), 3.19(3 \mathrm{H}, \mathrm{s}), 3.30(3 \mathrm{H}, \mathrm{s})$, $3.36(3 \mathrm{H}, \mathrm{s}), 3.42\left(1 \mathrm{H}, \mathrm{dd}, J \frac{1}{4} 3.3,10.4 \mathrm{~Hz}\right), 3.45(3 \mathrm{H}, \mathrm{s})$, $3.52(1 \mathrm{H}, \mathrm{dd}, J 1 / 47.2,10.4 \mathrm{~Hz}), 3.54(1 \mathrm{H}, \mathrm{d}, J 1 / 42.6 \mathrm{~Hz})$,

$3.65(1 \mathrm{H}$, ddd, $J 1 / 43.4,6.9,6.9 \mathrm{~Hz}), 3.75(1 \mathrm{H}$, ddd, $J 1 / 46.4$, $8.8,8.8 \mathrm{~Hz}), 4.05(1 \mathrm{H}$, ddd, $J / 45.8,8.7,8.7 \mathrm{~Hz}), 4.16(1 \mathrm{H}$, br d, J1/45.6 Hz); ${ }^{13} \mathrm{C}$ NMR $31.3\left(\mathrm{CH}_{2}\right), 48.4\left(\mathrm{CH}_{3}\right), 57.8$ $\left(\mathrm{CH}_{3}\right), 58.1\left(\mathrm{CH}_{3}\right), 59.1\left(\mathrm{CH}_{3}\right), 66.4\left(\mathrm{CH}_{2}\right), 72.1\left(\mathrm{CH}_{2}\right)$, $72.3(\mathrm{CH}), 76.5(\mathrm{CH}), 76.9(\mathrm{CH}), 77.2(\mathrm{CH}), 105.1(\mathrm{C})$; MS (EI) $m / z$ (rel intensity) $262\left(\mathrm{M}^{+}, 17\right), 231$ (1), 217 (23); HRMS calcd for $\mathrm{C}_{12} \mathrm{H}_{22} \mathrm{O}_{6}$ 262.1416, found 262.1379. Anal. Calcd for $\mathrm{C}_{12} \mathrm{H}_{22} \mathrm{O}_{6}$ : C, 54.95; $\mathrm{H}, 8.45$.

Found: C, 55.27; H, 8.10.

4.3.5. 1,3,4,5-Tetra- $O$-acetyl-2,8-anhydro-7deoxy-b- D-altro-oct-2-ulopyranose (30). Following the general procedure, precursor 10 (75 $\mathrm{mg}, 0.2 \mathrm{mmol})$ afforded the an- hydrosugar 30 (36 mg, $0.096 \mathrm{mmol}, 48 \%)$ as a colourless

oil: [a] $]_{\mathrm{D}}-36.9(c, 0.36)$; IR 2966, 1747, 1434, 1372, 1225, $1056 \mathrm{~cm}^{-1}$; ${ }^{1} \mathrm{H}$ NMR $1.97(1 \mathrm{H}, \mathrm{m}), 1.99(3 \mathrm{H}, \mathrm{s})$, $2.07(3 \mathrm{H}, \mathrm{s}), 2.08(1 \mathrm{H}, \mathrm{m}), 2.11(3 \mathrm{H}, \mathrm{s}), 2.16(3 \mathrm{H}, \mathrm{s}), 3.92$ (1H, ddd, $J / 42.7,10.8,10.8 \mathrm{~Hz}), 3.93(1 \mathrm{H}, \mathrm{d}, J 1 / 412.0 \mathrm{~Hz})$, $3.98(1 \mathrm{H}, \mathrm{ddd}, J 1 / 44.7,11.2,11.2 \mathrm{~Hz}), 4.17(1 \mathrm{H}, \mathrm{d}$, $J / 412.0 \mathrm{~Hz}), 4.36(1 \mathrm{H}, \mathrm{ddd}, J 1 / 42.0,3.7,11.1 \mathrm{~Hz}), 5.25$ (1H, dd, $J / 42.1,3.9 \mathrm{~Hz}), 5.38(1 \mathrm{H}, \mathrm{d}, J 1 / 410.3 \mathrm{~Hz}), 5.66$ $(1 \mathrm{H}, \mathrm{dd}, J 1 / 43.9,10.3 \mathrm{~Hz}) ;{ }^{13} \mathrm{C}$ NMR $20.6\left(2 \times \mathrm{CH}_{3}\right), 20.7$ $\left(\mathrm{CH}_{3}\right), 20.9\left(\mathrm{CH}_{3}\right), 26.1\left(\mathrm{CH}_{2}\right), 57.7\left(\mathrm{CH}_{2}\right), 63.4\left(\mathrm{CH}_{2}\right)$, $67.2(\mathrm{CH}), 67.8(\mathrm{CH}), 69.6(\mathrm{CH}), 71.9(\mathrm{CH}), 97.7(\mathrm{C})$, 169.8 (C), 170.1 (C), 170.3 (C), 170.5 (C); MS (EI) $\mathrm{m} / \mathrm{z}$ (rel intensity) $375\left(\mathrm{M}^{+}+\mathrm{H}, 1\right), 331$ (2), 315 (14); HRMS calcd for $\mathrm{C}_{16} \mathrm{H}_{23} \mathrm{O}_{10} 375.1291$, found 375.1266. Anal. Calcd for $\mathrm{C}_{16} \mathrm{H}_{22} \mathrm{O}_{10}$ : C, 51.33; H, 5.92. Found: C, 51.48; H, 6.11. 4.3.6. Methyl 3,7-anhydro-2,8-dideoxy-5,6-di- $O$ methyl- a-L-manno-oct-4-ulofuranoside (31) and 2,8-anhydro- 1,7-dideoxy-3,4,5-tri- $O$-methyl-b-Laltro-oct-2-ulopyra- nose (35). Following the general procedure, precursor $8(63 \mathrm{mg}, 0.269 \mathrm{mmol})$ afforded the anhydrosugar $35(7 \mathrm{mg}$,

$0.03 \mathrm{mmol}$, 11\%), and anhydrosugar 31 (28 mg, 0.12 mmol, $44.5 \%)$. Compound 35: crystalline compound, mp 80.5-82.5 ${ }^{\circ} \mathrm{C}$; $[\mathrm{a}]_{\mathrm{D}}+97.8$ (c, 0.45); IR 2940, 2885, 2831, 1469, 1378, 1119, $1064 \mathrm{~cm}^{-1} ;{ }^{1} \mathrm{H}$ NMR $1.37(3 \mathrm{H}$, s), $1.58(1 \mathrm{H}$, dddd, $J 1 / 43.5,6.8,10.3,13.7 \mathrm{~Hz}), 2.04(1 \mathrm{H}$, dddd, $J 1 / 42.7,4.8,10.8,13.5 \mathrm{~Hz}), 3.25(1 \mathrm{H}, \mathrm{d}, J 1 / 49.2 \mathrm{~Hz})$, $3.43(1 \mathrm{H}, \mathrm{dd}, J \mathrm{l} / 42.1,3.8 \mathrm{~Hz}), 3.48(3 \mathrm{H}, \mathrm{s}), 3.51(3 \mathrm{H}, \mathrm{s})$, $3.58(3 \mathrm{H}, \mathrm{s}), 3.74(1 \mathrm{H}, \mathrm{ddd}, J / 42.7,6.7,11.2 \mathrm{~Hz}), 3.87$ (1H, dd, $J 1 / 43.8,9.2 \mathrm{~Hz}), 3.90(1 \mathrm{H}$, ddd, $J 1 / 44.7,11.0$, $11.0 \mathrm{~Hz}), 4.36(1 \mathrm{H}, \mathrm{ddd}, J 1 / 41.9,3.6,10.8 \mathrm{~Hz}) ;{ }^{13} \mathrm{C} \mathrm{NMR}$ $23.0\left(\mathrm{CH}_{3}\right), 25.7\left(\mathrm{CH}_{2}\right), 56.5\left(\mathrm{CH}_{2}\right), 57.9\left(\mathrm{CH}_{3}\right), 58.1$ $\left(\mathrm{CH}_{3}\right), 61.4\left(\mathrm{CH}_{3}\right), 66.9(\mathrm{CH}), 78.7(\mathrm{CH}), 80.1(\mathrm{CH}), 82.7$ (CH), 99.2 (C); MS (EI) $\mathrm{m} / \mathrm{z}$ (rel intensity) $232\left(\mathrm{M}^{+},<1\right)$,
217 (<1), 201 (2); HRMS calcd for $\mathrm{C}_{11} \mathrm{H}_{20} \mathrm{O}_{5} 232.1310$, found 232.1334. Anal. Calcd for $\mathrm{C}_{11} \mathrm{H}_{20} \mathrm{O}_{5}: \mathrm{C}, 56.88 ; \mathrm{H}$, 8.68. Found: C, 56.87; H, 8.58. Compound 31: colourless oil; [a $]_{\mathrm{D}}-8.4(c, 0.55)$; IR 2934, 2827, 1446, 1103, $1050 \mathrm{~cm}^{-1} ;{ }^{1} \mathrm{H}$ NMR $1.29\left(3 \mathrm{H}, \mathrm{d}, J \frac{1}{4} 6.7 \mathrm{~Hz}\right), 1.89(1 \mathrm{H}$, $\mathrm{m}), 2.35(1 \mathrm{H}, \mathrm{m}), 3.07(1 \mathrm{H}, \mathrm{dd}, J \mathrm{l} / 42.4,6.2 \mathrm{~Hz}), 3.26(3 \mathrm{H}$, s), $3.45(3 \mathrm{H}, \mathrm{m}), 3.52(3 \mathrm{H}, \mathrm{s}), 3.61(1 \mathrm{H}, \mathrm{d}, \mathrm{J} / 42.4 \mathrm{~Hz})$, $3.66(1 \mathrm{H}, \mathrm{dq}, J 1 / 46.2,6.7 \mathrm{~Hz}), 3.82(1 \mathrm{H}$, ddd, $J 1 / 46.4,8.8$, $8.8 \mathrm{~Hz}), 4.11\left(1 \mathrm{H}_{13} \mathrm{ddd}, J / 45.5,8.8,8.8 \mathrm{~Hz}\right), 4.21(1 \mathrm{H}, \mathrm{dd}$, $J 1 / 41.7,6.9 \mathrm{~Hz}) ;{ }^{13} \mathrm{C}$ NMR $18.6\left(\mathrm{CH}_{3}\right), 31.4\left(\mathrm{CH}_{2}\right), 48.5$ $\left(\mathrm{CH}_{3}\right), 57.9\left(\mathrm{CH}_{3}\right), 58.1\left(\mathrm{CH}_{3}\right), 66.4\left(\mathrm{CH}_{2}\right), 68.7(\mathrm{CH})$, $76.7(2 \times \mathrm{CH}) ; \mathrm{MS}(\mathrm{EI}) \mathrm{m} / \mathrm{z}$ (rel intensity) $232(\mathrm{M}, 16)$, 217 (1), 201 (4), 185 (2), 169 (2); HRMS calcd for $\mathrm{C}_{11} \mathrm{H}_{20} \mathrm{O}_{5}$ 232.1310, found 232.1282. Anal. Calcd for $\mathrm{C}_{11} \mathrm{H}_{20} \mathrm{O}_{5}$ : C, 56.88; H, 8.68. Found: C, 57.08; H, 8.32. 4.3.7. Formation of compounds 31,35 and $3-$ acetyl-2,8- anhydro-1,7-didesoxy-3,4,5-tri- $O$ methyl-L-arabino- octo-2,5-diulo-2,6-pyranose (36). Following the general procedure for IHA reactions adding $\mathrm{NaHCO}_{3}(100 \%)$ to the reaction mixture, precursor 8 (360 mg, $1.53 \mathrm{mmol})$ af- forded anhydrosugars 35 (50 $\mathrm{mg}, 0.215 \mathrm{mmol}, 14 \%), 31$ (130 mg, $0.56 \mathrm{mmol}, 37 \%)$ and 36 (20 mg, $0.07 \mathrm{mmol}, 4.5 \%)$. Compound 36: $[\mathrm{a}]_{\mathrm{D}}+11.1$ $(c, 0.28)$; IR 2943, 2747, 1222, $1112 \mathrm{~cm}^{-1}$; ${ }^{1} \mathrm{H}$ NMR 1.35 (3H, s), 1.77 (1H, dddd,

$J 1 / 43.7,6.8,10.6,14.2 \mathrm{~Hz}), 1.99(1 \mathrm{H}$, dddd, $J 1 / 42.8,4.0$, 10.5, 14.0 Hz), $2.10(3 \mathrm{H}, \mathrm{s}), 3.08(1 \mathrm{H}, \mathrm{d}, J / 48.9 \mathrm{~Hz}), 3.59$ $(3 \mathrm{H}, \mathrm{s}), 3.62(3 \mathrm{H}, \mathrm{s}), 3.66(3 \mathrm{H}, \mathrm{s}), 3.72(1 \mathrm{H}, \mathrm{ddd}, \mathrm{J} / \mathrm{4} 2.5$, $6.7,11.2 \mathrm{~Hz}), 3.82(1 \mathrm{H}$, ddd, J1/44.4, $10.8,10.8 \mathrm{~Hz}), 3.85$ $(1 \mathrm{H}, \mathrm{d}, J 1 / 48.9 \mathrm{~Hz}), 5.21(1 \mathrm{H}, \mathrm{dd}, J 1 / 43.8,10.6 \mathrm{~Hz}),{ }^{13} \mathrm{C}$ NMR $21.8\left(\mathrm{CH}_{3}\right), 22.7\left(\mathrm{CH}_{3}\right), 24.5\left(\mathrm{CH}_{2}\right), 53.3\left(\mathrm{CH}_{3}\right)$, $56.0\left(\mathrm{CH}_{2}\right), 61.6\left(\mathrm{CH}_{3}\right), 61.8\left(\mathrm{CH}_{3}\right), 67.1(\mathrm{CH}), 83.3(\mathrm{CH})$, 84.4 (CH), 98.5 (C), 105.0 (C), 169.6 (C); MS (EI) $\mathrm{m} / \mathrm{z}$ (rel intensity) $290\left(\mathrm{M}^{+}, 3\right), 259$ (7), 231 (6), 199 (8); HRMS calcd for $\mathrm{C}_{13} \mathrm{H}_{22} \mathrm{O}_{7} 290.1365$, found 290.1314 . Anal. Calcd for $\mathrm{C}_{13} \mathrm{H}_{22} \mathrm{O}_{7}$ : C, 53.78; H, 7.64. Found: C, 53.80; H, 7.39.

4.3.8.

dideoxy-b-L-

3,4,5-Tri-O-acetyl-2,8-anhydro-1,7altro-oct-2-ulopyranose (32).

Following the general pro- cedure, precursor 6 (52 $\mathrm{mg}$, $0.163 \mathrm{mmol})$ afforded the anhydrosugar $32(27 \mathrm{mg}, 0.085$ mmol, 53\%) as a colourless oil: $[\mathrm{a}]_{\mathrm{D}}+65(c, 0.36)$; IR 2972, 2747, 1372, 1226, $1059 \mathrm{~cm}^{-1}$; ${ }^{1} \mathrm{H}$ NMR $1.31(3 \mathrm{H}, \mathrm{s})$, $1.87(1 \mathrm{H}, \mathrm{m}), 1.98(3 \mathrm{H}, \mathrm{s}), 2.09(3 \mathrm{H}, \mathrm{s}), 2.11(1 \mathrm{H}, \mathrm{m})$, $2.14(3 \mathrm{H}, \mathrm{s}), 3.83-3.94(2 \mathrm{H}$,

m), 4.25 (1H, ddd, $J 1 / 42.2,2.2,10.8 \mathrm{~Hz}), 5.21(1 \mathrm{H}, \mathrm{d}$, $J 1 / 49.9 \mathrm{~Hz}), 5.23(1 \mathrm{H}, \mathrm{dd}, J 1 / 41.9,3.9 \mathrm{~Hz}), 5.63(1 \mathrm{H}, \mathrm{dd}$, $J 1 / 43.9,9.9 \mathrm{~Hz}) ;{ }^{13} \mathrm{C} \mathrm{NMR} 20.6\left(\mathrm{CH}_{3}\right), 20.8\left(\mathrm{CH}_{3}\right), 20.9$ $\left(\mathrm{CH}_{3}\right), 22.9\left(\mathrm{CH}_{3}\right), 26\left(\mathrm{CH}_{2}\right), 56.8\left(\mathrm{CH}_{2}\right), 67.4(\mathrm{CH}), 69.4$ $(\mathrm{CH}), 71.7(\mathrm{CH}), 72.2(\mathrm{CH}), 98.7(\mathrm{C}), 170.1(\mathrm{C}), 170.2$ (C), 170.5 (C); MS (EI) m/z (rel intensity) $316\left(\mathrm{M}^{+}, 7\right), 273$ (1), 257 (10), 197 (7), 155 (25); HRMS calcd for $\mathrm{C}_{14} \mathrm{H}_{20} \mathrm{O}_{8} 316.1158$, found 316.1189. Anal. Calcd for $\mathrm{C}_{14} \mathrm{H}_{20} \mathrm{O}_{8}$ : C, 53.16; H, 6.37. Found: C, 53.23; H, 6.10.

4.3.9. (2R)-2,8-Anhydro-5,7-dideoxy-1,3,4-tri- $O$ methyl- D-arabino-oct-2-ulopyranose (33). Following the general procedure, precursor 17 (140 mg, $0.60 \mathrm{mmol})$ afforded the cyclised compound $33(88.3 \mathrm{mg}$, $0.38 \mathrm{mmol}, 64 \%$ ) as a colourless oil: [a $]_{\mathrm{D}}-3.3(c, 0.09)$; IR 2931, 2830, 1731, 1450, 1260, 1195, $1113 \mathrm{~cm}^{-1}$; ${ }^{1} \mathrm{H}$ NMR $1.62(1 \mathrm{H}, \mathrm{m}), 1.83(1 \mathrm{H}$, $\mathrm{m}), 2.04(1 \mathrm{H}, \mathrm{m}), 2.17(1 \mathrm{H}, \mathrm{m}), 3.29-3.37(2 \mathrm{H}, \mathrm{m}), 3.39$ $(3 \mathrm{H}, \mathrm{s}), 3.45(1 \mathrm{H}, \mathrm{m}), 3.45(3 \mathrm{H}, \mathrm{s}), 3.55(3 \mathrm{H}, \mathrm{s}), 3.75-3.86$ 
(2H, m), $3.91(1 \mathrm{H}, \mathrm{m}), 4.34(1 \mathrm{H}, \mathrm{m}) ;{ }^{13} \mathrm{C} \mathrm{NMR} 28.9\left(\mathrm{CH}_{2}\right)$, $35.8\left(\mathrm{CH}_{2}\right), 57.4\left(\mathrm{CH}_{3}\right), 58.2\left(\mathrm{CH}_{2}\right), 59.3\left(\mathrm{CH}_{3}\right), 60.8\left(\mathrm{CH}_{3}\right)$, $66.3(\mathrm{CH}), 73.2\left(\mathrm{CH}_{2}\right), 78.1(\mathrm{CH}), 81.7(\mathrm{CH}), 98.5(\mathrm{C})$; MS $\mathrm{m} / \mathrm{z}$ (rel intensity) $232\left(\mathrm{M}^{+}, 1\right), 231$ (2); HRMS calcd for $\mathrm{C}_{11} \mathrm{H}_{20} \mathrm{O}_{5}$ 232.1310, found 232.1297. Anal. Calcd for $\mathrm{C}_{11} \mathrm{H}_{20} \mathrm{O}_{5}$ : C, 56.88; H, 8.68. Found: C, 56.81; H, 8.90. 4.3.10.

3,6-Anhydro-2-deoxy-7-O(methoxymethyl)-4,5- $O$-methylene-b-D-ribo-hept4-ulofuranose (34). Follow- ing the general procedure, precursor $21(66.2 \mathrm{mg}$,

$0.28 \mathrm{mmol})$ afforded the cyclised compound $34(37.4 \mathrm{mg}$, $0.16 \mathrm{mmol}, 57 \%)$ as a colourless oil: $[\mathrm{a}]_{\mathrm{D}}+61.0(c, 1.41)$; IR 2930, 2886, 1732, 1440, 1367, 1153, $1109 \mathrm{~cm}^{-1} ;{ }^{1} \mathrm{H}$ NMR 1.99-2.17 (2H, m), $3.37(3 \mathrm{H}, \mathrm{s}), 3.70(1 \mathrm{H}, \mathrm{dd}$, $J 1 / 46.4,10.7 \mathrm{~Hz}), 3.76(1 \mathrm{H}, \mathrm{dd}, J / 44.0,10.7 \mathrm{~Hz}), 4.04-4.10$ $(2 \mathrm{H}, \mathrm{m}), 4.18-4.22(2 \mathrm{H}, \mathrm{m}), 4.39(1 \mathrm{H}, \mathrm{dd}, J 1 / 41.4,5.7 \mathrm{~Hz})$, $4.67(2 \mathrm{H}, \mathrm{s}), 5.25(2 \mathrm{H}, \mathrm{s}), 5.34(2 \mathrm{H}, \mathrm{s}) ;{ }^{13} \mathrm{C}$ NMR 31.1 $\left(\mathrm{CH}_{2}\right), 55.4\left(\mathrm{CH}_{3}\right), 67.3\left(\mathrm{CH}_{2}\right), 69.5\left(\mathrm{CH}_{2}\right), 82.5(\mathrm{CH})$, $84.0(\mathrm{CH}), 84.2(\mathrm{CH}), 96.6\left(\mathrm{CH}_{2}\right), 98.4\left(\mathrm{CH}_{2}\right), 123.2(\mathrm{C})$; MS $m / z$ (rel intensity) $232\left(\mathrm{M}^{+}, 2\right), 231$ (7); HRMS calcd for $\mathrm{C}_{10} \mathrm{H}_{16} \mathrm{O}_{6}$ 232.0947, found 232.0991. Anal. Calcd for $\mathrm{C}_{10} \mathrm{H}_{16} \mathrm{O}_{6}$ : C, 51.72; H, 6.94. Found: C, 51.62; H, 6.88.

4.3.11. (4R)-3,6-Anhydro-2-deoxy-4,5:7,8-di-Oisopropyl- idene-a-D-manno-oct-4-ulofuranose (37) and 7-deoxy- 1,2:4,5-di- $O$-isopropylideneD-altro-oct-3-ulofuranose (38). Following the general procedure, precursor $23(90 \mathrm{mg}$,

$0.31 \mathrm{mmol})$ afforded compounds 38 (23 mg, $0.08 \mathrm{mmol}$, $26 \%$ ) and 37 (36 mg, $0.13 \mathrm{mmol}, 41 \%$ ). Compound 37:

amorphous solid; [a] $]_{\mathrm{D}}-19.0(c, 0.5)$; IR 2988, 2891,

1374, 1210, $1085 \mathrm{~cm}^{-1} ;{ }^{1} \mathrm{H}$ NMR $1.34(3 \mathrm{H}, \mathrm{s}), 1.42(3 \mathrm{H}$, s), $1.46(3 \mathrm{H}, \mathrm{s}), 1.48(3 \mathrm{H}, \mathrm{s}), 1.93(1 \mathrm{H}, \mathrm{m}), 2.13(1 \mathrm{H}, \mathrm{m})$, $4.04(4 \mathrm{H}, \mathrm{m}), 4.13(1 \mathrm{H}, \mathrm{dd}, J 1 / 43.9,7.3 \mathrm{~Hz}), 4.35$ (1H, ddd, $J 1 / 44.8,6.3,7.3 \mathrm{~Hz}), 4.45(1 \mathrm{H}, \mathrm{d}, J 1 / 43.9 \mathrm{~Hz}), 4.54(1 \mathrm{H}, \mathrm{dd}$, $J 1 / 47.1,7.1 \mathrm{~Hz}) ;{ }^{13} \mathrm{C}$ NMR $25.0\left(\mathrm{CH}_{3}\right), 25.1\left(\mathrm{CH}_{3}\right), 26.6$ $\left(\mathrm{CH}_{3}\right), 26.8\left(\mathrm{CH}_{3}\right), 30.8\left(\mathrm{CH}_{2}\right), 66.4\left(\mathrm{CH}_{2}\right), 69.0\left(\mathrm{CH}_{2}\right)$, $73.1(\mathrm{CH}), 80.3(\mathrm{CH}), 83.5(\mathrm{CH}), 86.4(\mathrm{CH}), 109.1(\mathrm{C})$, 114.0 (C), 122.8 (C); MS m/z (rel intensity) 271 $\left(\mathrm{M}^{+}-\mathrm{CH}_{3}, 35\right), 213$ (30); HRMS calcd for $\mathrm{C}_{13} \mathrm{H}_{19} \mathrm{O}_{6}$ 271.1181, found 271.1192. Anal. Calcd for $\mathrm{C}_{14} \mathrm{H}_{22} \mathrm{O}_{6}$ : C, 58.73; H, 7.74. Found: C, 58.85; H, 7.95. Compound 38: amorphous solid; [a $]_{\mathrm{D}}-24.1(c, 0.8)$; IR 2986, 2873,

1732, 1378, 1260, 1209, $1077 \mathrm{~cm}^{-1} ;{ }^{1} \mathrm{H}$ NMR $1.34(1 \mathrm{H}$, m), $1.34(3 \mathrm{H}, \mathrm{s}), 1.36(3 \mathrm{H}, \mathrm{s}), 1.43(3 \mathrm{H}, \mathrm{s}), 1.46(3 \mathrm{H}, \mathrm{s})$, $2.19(1 \mathrm{H}, \mathrm{m}), 3.70(1 \mathrm{H}$, ddd, $J 1 / 44.2,12.5,12.5 \mathrm{~Hz}), 3.98$ $(1 \mathrm{H}$, ddd, $J 1 / 40.0,6.7,12.6 \mathrm{~Hz}), 4.03(1 \mathrm{H}, \mathrm{dd}, J 1 / 47.5$, $7.5 \mathrm{~Hz}), 4.20(1 \mathrm{H}, \mathrm{dd}, J 1 / 45.6,8.4 \mathrm{~Hz}), 4.33$ (1H, ddd, $J 1 / 40.0,6.0,6.0 \mathrm{~Hz}), 4.36(1 \mathrm{H}, \mathrm{m}), 4.68(1 \mathrm{H}, \mathrm{d}, J 1 / 45.6 \mathrm{~Hz})$, $4.73(1 \mathrm{H}, \mathrm{d}, J 1 / 45.6 \mathrm{~Hz}) ;{ }^{13} \mathrm{C}$ NMR $24.4\left(\mathrm{CH}_{3}\right), 25.0\left(\mathrm{CH}_{3}\right)$, $25.8\left(\mathrm{CH}_{3}\right), 25.9\left(\mathrm{CH}_{3}\right), 27.5\left(\mathrm{CH}_{2}\right), 59.9\left(\mathrm{CH}_{2}\right), 64.9$ $\left(\mathrm{CH}_{2}\right), 74.7(\mathrm{CH}), 79.3(\mathrm{CH}), 82.2(\mathrm{CH}), 82.8(\mathrm{CH})$, 105.6(C), 109.7 (C), 112.5 (C); MS m/z (rel intensity) 271 $\left(\mathrm{M}^{+}-\mathrm{CH}_{3}, 47\right), 213$ (86); HRMS calcd for $\mathrm{C}_{13} \mathrm{H}_{19} \mathrm{O}_{6}$ 271.1181, found 271.1212. Anal. Calcd for $\mathrm{C}_{14} \mathrm{H}_{22} \mathrm{O}_{6}: \mathrm{C}$, 58.73; H, 7.74. Found: C, 58.63; H, 7.70.

4.3.12. 7-Deoxy-1,2:4,5-di-O-oxomethylene-D-altrooct-3- ulofuranose (40). A solution of the alcohol 26 (50 mg,

$0.19 \mathrm{mmol}$ ) in dry $\mathrm{CH}_{2} \mathrm{Cl}_{2}(4 \mathrm{~mL})$ containing DIB (93 mg, $0.29 \mathrm{mmol}$ ) and iodine (49 $\mathrm{mg}, 0.19 \mathrm{mmol}$ ) was stirred and irradiated with an $80 \mathrm{~W}$ tungsten filament lamp at room temperature under nitrogen atmosphere for $35 \mathrm{~min}$. The reaction mixture was then poured into $10 \%$ aqueous $\mathrm{Na}_{2} \mathrm{~S}_{2} \mathrm{O}_{3}$ and extracted with EtOAc. The organic phase was concentrated in vacuum. The residue was dissolved in dry $\mathrm{CH}_{2} \mathrm{Cl}_{2}$ and $\mathrm{BF}_{3} \$ \mathrm{OEt}_{2}(24 \mathrm{~mL}, 0.19 \mathrm{mmol})$ was added at $0{ }^{\circ} \mathrm{C}$ under nitrogen atmosphere. After $30 \mathrm{~min}$ at $0{ }^{\circ} \mathrm{C}$, the reaction mixture was poured into aqueous sat. solution of $\mathrm{NaHCO}_{3}$ and extracted with AcOEt. The organic phase was dried with $\mathrm{Na}_{2} \mathrm{SO}_{4}$ and concentrated under vacuum. Column chromatography of the residue (hexanes-EtOAc, 1:1) afforded the anhydrosugar $40(32 \mathrm{mg}, 0.12 \mathrm{mmol}, 65 \%)$ as a colourless crystalline compound; [a] $]_{\mathrm{D}}-42.6(c, 0.6$, EtOAc $) ; \mathrm{mp}$ 209-213 ${ }^{\circ} \mathrm{C}($ EtOAc-n-pentane $) ;{ }^{1} \mathrm{H}$ NMR 1.60 (1H, dddd, $J 1 / 40.8,2.1,3.9,14.1 \mathrm{~Hz}), 2.38$ (1H, dddd, J1/43.9, 6.9, $13.0,14.0 \mathrm{~Hz}), 3.71(1 \mathrm{H}$, ddd, J1/43.9, $12.9,12.9 \mathrm{~Hz}), 4.12$ (1H, dddd, $J 1 / 41.1,1.1,6.8,12.5 \mathrm{~Hz}), 4.52(1 \mathrm{H}, \mathrm{dd}, J 1 / 48.6$, $8.6 \mathrm{~Hz}), 4.70(1 \mathrm{H}, \mathrm{ddd}, J \mathrm{l} / 41.6,1.6,3.5 \mathrm{~Hz}), 4.73(1 \mathrm{H}, \mathrm{dd}$, $J 1 / 44.5,8.7 \mathrm{~Hz}), 4.88\left(1 \mathrm{H}, \mathrm{dd}, J \frac{1}{4} 4.5,8.5 \mathrm{~Hz}\right), 5.20(1 \mathrm{H}, \mathrm{d}$, $J / 46.1 \mathrm{~Hz}), 5.26(1 \mathrm{H}, \mathrm{d}, J 1 / 46.1 \mathrm{~Hz}) ;{ }^{13} \mathrm{C}$ NMR $27.0\left(\mathrm{CH}_{2}\right)$, $60.6\left(\mathrm{CH}_{2}\right), 65.2\left(\mathrm{CH}_{2}\right), 73.2(\mathrm{CH}), 80.3(\mathrm{CH}), 81.2(\mathrm{CH})$, $81.3(\mathrm{CH}), 104.5(\mathrm{C}), 153.5(\mathrm{C}), 154.8(\mathrm{C}) ; \mathrm{MS}\left(\mathrm{FAB}^{+}\right)$ $\mathrm{m} / \mathrm{z}$ (rel intensity) $281\left(\mathrm{M}^{+}+\mathrm{Na}, 18\right), 259\left(\mathrm{M}^{+}+\mathrm{H}, 100\right)$; HRMS calcd for $\mathrm{C}_{10} \mathrm{H}_{11} \mathrm{O}_{8} 259.0454$, found 259.0445 . Anal. Calcd for $\mathrm{C}_{10} \mathrm{H}_{10} \mathrm{O}_{8}$ : C, 46.52; H, 3.90. Found: C, 46.19; H, 4.20.

\section{Acknowledgements}

This work was supported by the investigation programmes CTQ2004-06381/BQU and CTQ20004-02367/BQU of the Direcci' on General de Investigaci' on Cient ' Ifica y T' ecnica, Spain. A.J.H. and I.P.-M. thank the Ministerio de Educaci' on y Ciencia, Spain, and the programme I3P-CSIC, respectively, for fellowships.

\section{References and notes}

1. Recent reviews: (a) Majetich, G. Tetrahedron 1995, 51, 7095- 7129; (b) Feray, L.; Kuznersov, N.; Renaud, P. Radicals in Organic Synthesis; Renaud, P., Sibi, M. P., Eds.; WileyVCH: Weinheim, 2001; Vol. 2, pp 246-278; (c) Robertson, J.; Pillai, J.; Lush, R. K. Chem. Soc. Rev. 2001, 30, 94-103.

2. (a) Mart' in, A.; Salazar, J. A.; Su' arez, E. J. Org. Chem. 1996, 61, 3999-4006; (b) Dorta, R. L.; Mart' in, A.; Salazar, J. A.; Su' arez,

E. J. Org. Chem. 1998, 63, 2251-2261; (c) Francisco, C. G.;

Herrera, A. J.; Su' arez, E. J. Org. Chem. 2002, 67, 7439-7445; For a preliminary communication on this work, see: (d) Francisco, C. G.; Freire, R.; Herrera, A. J.; P' erez-Mart' in, I.; Su'arez, E. Org. Lett. 2002, 4, 1959-1961; For other IHA reactions in carbohydrate chemistry, see: (e) Descotes, G. J. Carbohydr. Chem. 1988, 7, 1-20; (f) Chatgilialoglu, C.; Giminis, T.; Spada, G. P. Chem.-Eur. J. 1999, 5, 28662876;

(g) Robins, M. J.; Guo, Z.; Samano, M. C.; Wnuk, S. F. J. Am. Chem. Soc. 1999, 121, 1425-1433.

3. (a) Paquete, L. A.; Sun, L. Q.; Friedrich, D.; Savage, P. B. J. Am. Chem. Soc. 1997, 119, 8438-8450; (b) Bruke, S. D.; Kort, M. E.; Strickland, S. M. S.; Organ, H. M.; Silks, L. A. Tetrahedron Lett. 1994, 35, 1503-1506; (c) Hatakeyama, S.; Kawamura, M.; Takano, S. J. Am. Chem. Soc. 1994, 116, 4081-4082.

4. (a) Danishefsky, S. J.; Armistead, D. A.; Wincott, F. E.; Selnick, H. G.; Hungate, R. J. Am. Chem. Soc. 1989, 111, 2967-2980; (b) Brimble, M. A.; Far`es, F. A. Tetrahedron 
1999, 55, 7661-7706; (c) Concepci' on, J. I.; Francisco, C. G.; Hern' andez, R.; Salazar, J. A.; Su'arez, E. Tetrahedron Lett. 1984, 25, 1953-1984.

5. HAT reaction through eight-membered transition states or higher promoted by alkoxyl radicals which suffer a severe entropic penalty are practically unknown. Recently an IHA through a nine-membered transition state between glucopyranose units in a disaccharide model has been observed in this laboratory: Francisco, C. G.; Herrera, A. J.; Kennedy, A. R.; Meli'an, D.; Su'arez, E. Angew. Chem., Int. Ed. 2002 , $41,860-862$.

6. CRC Handbook of Chemistry and Physics, 73rd ed.; CRC: Boca Raton, FL, 1992; pp 9-138.

7. (a) Gonz' alez, C. C.; Kennedy, A. R.; Le'on, E. I.; RiescoFagundo, C.; Su' arez, E. Angew. Chem., Int. Ed. 2001, 40, 2326-2328; (b) Francisco, C. G.; Gonz'alez, C. C.; Su' arez, E. J. Org. Chem. 1998, 63, 8092-8093.

8. An example of the influence of the substituents on the 1,5-HAT reaction compared with competing b-fragmentation of alkoxyl radicals has been published: Allen, P. R.; Brimble, M. A.; Far'es, F. A. J. Chem. Soc., Perkin Trans. 1 1998, 2403-2411.

9. No products arising from the geometrically possible abstraction of the hydrogen at C5 have been detected in the examples described in this work. Probably the abstraction at this position is disfavoured by steric and stereoelectronic factors. For studies of stereoelectronic effects in intramolecular hydrogen abstraction reactions, see: (a) Malatesta, V.; Ingold, K. U. J. Am. Chem. Soc. 1981, 103, 609-614; (b) Beckwith, A. L. J.; Easton, C. J. J. Am. Chem. Soc. 1981, 103, 615-619.

10. For competition between 1-5-, 1-6- and 1-7-hydrogen transfer, see: Denenmark, D.; Winkler, T.; Waldner, A.; De Mesmaeker, A. Tetrahedron Lett. 1992, 33, 3613-3616.

11. (a) Satake, M.; Ofuji, K.; Naoki, H.; James, K. J.; Furey, A.; McMahon, T.; Silke, J.; Yasumoto, T. J. Am. Chem. Soc. 1998, 120, 9967-9968; (b) Aiguade, J.; Hao, J.; Forsyth, C. J. Org. Lett. 2001, 3, 979-982; (c) Nicolaou, K. C.;

Oihko, P. N.; Diedrichs, N.; Zou, N.; Bernal, F. Angew. Chem., Int. Ed. 2001, 40, 1262-1265.

12. (a) Li, X.-L.; Cheng, X.; Yang, L. M.; Wang, R.-R.; Zheng, Y.-T.; Xiao, W.-L.; Zhao, Y.; Xu, G.; Lu, Y.; Chang, Y.; Zheng, Q.-T.; Zhao, Q.-S.; Sun, H.-D. Org. Lett. 2006, 8, 1937-1940; (b) Yamada, M.; Hayashi, K.-I.; Ikeda, S.; Tsutsui, K.; Tsutsui, K.; Ito, T.; Iinuma, M.; Nozaki, H. Biol. Pharm. Bull. 2006, 29, 1504-1507; (c) Perlmutter, P.;
Selajerern, W.; Vounatsos, F. Org. Biomol. Chem. 2004, 2, 2220-2228.

13. (a) Luengo, J. I.; Gleason, J. G. Tetrahedron Lett. 1992, 33, 6911-6914; (b) Richter, P. K.; Tomaszewski, M. J.; Miller, R. A.; Patron, A. P.; Nicolaou, K. C. J. Chem. Soc., Chem. Commun. 1994, 1151-1152.

14. Evidence for a deactivating influence of the b-oxygen on the intermolecular hydrogen abstraction reaction has been described: (a) Busfield, W. K.; Grice, I. D.; Jenkins, I. D.; Monteiro, M. J. J. Chem. Soc., Perkin Trans. 2 1994, 1071-1077; (b) Busfield,

W. K.; Grice, I. D.; Jenkins, I. D.; Monteiro, M. J. J. Chem. Soc., Perkin Trans. 2 1994, 1079-1086.

15. An example of a double IHA reaction:

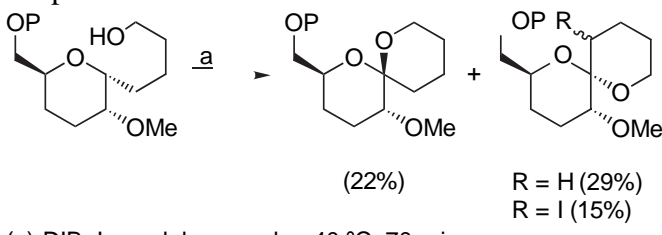

(a) DIB, $\mathrm{I}_{2}$, cyclohexane, $\mathrm{h} v, 40^{\circ} \mathrm{C}, 70 \mathrm{~min}$

For other double IHA reactions in carbohydrate models, see Ref. 2a, $\underline{b}$.

16. For studies of the influence of polar factors on, see: (a) Beckwith, A. L. J.; Zavitsas, A. A.; Chatgilialoglu, C. J. Am. Chem. Soc. 1995, 117, 10645-10654; (b) Kaushal, P.; Mok, P. L. H.; Roberts, B. P. J. Chem. Soc., Perkin Trans. 2 1990, 1663-1670. For an example of correlation between proximity in the ground state and regioselectivity in an IHA reaction, see Ref. $\underline{3 b}$.

17. Bicyclic ketals can be easily converted into the corresponding bicyclic ethers by stereoselective reduction: (a) Takemoto, Y.; Furuse, S.-I.; Hayase, H.; Echigo, T.; Iwata, C.; Tanaka, T.; Ibuka, T. Chem. Commun. 1999, 2515-2516; (b) Nicolaou,

K. C.; Hwang, C.-K.; Nugiel, D. A. J. Am. Chem. Soc. 1989 , $111,4136-4137$.

18. Giannis, A.; Sandhoff, K. Tetrahedron Lett. 1985, 26, 1479 1482.

19. Miljkovic, M.; Habash-Marino, M. J. Org. Chem. 1983, 48, 855-860.

20. Beckwith, A. L. J.; Duggan, P. J. J. Chem. Soc., Perkin Trans. 2 1993, 1673-1680.

21. Francisco, C. G.; Herrera, A. J.; Su' arez, E. J. Org. Chem. 2003 , $68,1012-1017$. 